\title{
Untangling the Mathematical Relation Between Natural Selection and Adaptive Radiation Using Macromolecules and Microevolutionary Forces
}

\author{
Bharat Kwatra ${ }^{1 *}$, Maanvi Mudgil ${ }^{*}$ \\ State University of New York ${ }^{1}$, University of Delhi ${ }^{2}$.
}

\section{ABSTRACT}

Simulating natural selection over subsequent generations of Oniscus asellus, the fittest selected male and female bugs in different diet was used to analyze the concentration of proteins in their body along their frequency in continuous mapped generations over hundred seed values, further DNA Fingerprinting of these selected bugs revealed a relationship with their parents originated from different geographic areas/borders. It was observed that variation in microevolutionary forces caused variation in macromolecules by setting up a geneenvironment interaction which deduced natural selection in order to define adaptive radiation and speciation by micro-evolutionary forces.

Keywords : Pill Bugs, Evolution, Adaptive Radiation, DNA Fingerprinting, Natural Selection, Regression Analysis, Gene-environment interaction, Genetic Drift, Macromolecules, Hardy Weinberg Principle, Isopoda

\section{INTRODUCTION}

Oniscus asellus, commonly known as woodlouse, is a crustacean which belongs to monophyletic suborder Oniscidea within the isopods. They generally grow to a length of $16 \mathrm{~mm}$ and a width of $6 \mathrm{~mm}$. They have a basic morphology of a segmented, dorsoventrally flattened body with paired jointed legs and specialized appendages for respiration. Due to the presence of their varied forms, relatively short duration of reproduction, conglobating abilities and ease of handling they become a suitable choice for performing the experiment. Microevolution is defined as the change in allele frequencies that occurs over a relatively short (evolutionary terms) duration of time within a population. This happens as a result of different processes namely mutation, selection, genetic drift, gene flow etc. There exists variation in the population of organisms which makes the fittest one to survive elucidated in the theory of Natural selection explained by Darwin, is a key mechanism of evolution. In natural selection, those variations in the genotype that increase an organism's chances of survival and procreation are preserved and multiplied from generation to generation at the expense of less advantageous ones. Mutation theory, ideate that new species are formed from the sudden and unexpected emergence of alterations in their defining traits by Dutch botanist and geneticist Hugo de Vries was opposed by the scientific committee and the concept of Natural selection with linkage and recombination was introduced in a new theory called Neo Darwinism based on the work of Charles Darwin and T.H. Morgan respectively . Based on "Neo Darwinism", Theory of Adaptive radiation was introduced and is defined as a process in which organisms diversify rapidly from an ancestral species into a number of other forms. This diversification may have occurred due to a change in the environment which may bring about changes in resource availability. The experiment involves understanding the link between microevolutionary forces and adaptive radiation by simulating natural selection over generations of Oniscus asellus, in order 
to establish link b/w Mutation theory, Neo Darwinism and adaptive radiation, which was done by identifying the fittest bugs over ten subsequent generations using random correlated selection on the basis of parameters like length, weight, age, average speed, diet etc. under the attack of different predation tools namely fork, spoon, chopsticks, scapula and tweezers. The relation between different parameters with average speed was used for analyzing the trend under which the bugs had maximum average speed thereby maximum survival. The selected male and female bugs under two different diets were further studied to understand which microevolutionary forces operate over the generations. An analysis between simulated continuous mapped generations over hundred seed values was done to identify the microevolutionary forces. The frequency of selected bugs exhibited a genetic drift trend. Mutation and selection trends were exhibited by $A_{1} A_{1}$ and $A_{2} A_{2}$ in males whereas mutation and selection trends were exhibited by $A_{1} A_{2}$ in females. Analyzing the frequency of occurrence of bugs over continuous mapping for hundred seed values the male and female bugs under different diets having a maximum frequency, where selected and were used to perform the Bovine Serum Albumin (BSA) Assay Test, in order to identify the protein level in each individual. It was observed that bugs with higher frequency would have maximum protein i.e. generation by generation protein synthesis in the body of the organism increased as per environmental factors received, which establishes the gene-environment relation and sudden mutation. Further, the genetic data of the fittest bugs of different origin in a parental generation was compared to that of fittest bugs selected from filial generations and adaptive radiation was identified using DNA mapping/fingerprinting techniques.

\section{METHODS AND MATERIAL}

A total of thousand bugs were bought in for the experiment, out of the total 500 bugs were of Portuguese origin and 500 of Ukrainian origin. [1] The first experiment was performed by randomly choosing 50 of these bugs, 25 males, and 25 females which were collected in a glass. The setup included 12 inches by 12 inches box [fig 1.1]. All the four corners had triangular closings of side 3 inches extending on both adjacent sides of the box. A circle with a radius of 2 inches was marked in the center of the box. The box was filled with moist and damp mud and had randomly placed twigs in it. [2] The glass containing the bugs was inverted over the center of the setup and bugs were gently tapped and a time of 1 minute was given for settling post which the glass was removed. After 5 seconds the bugs were removed one at a time using the predation tool. The process continued until all the bugs had hidden or had been removed. [3] Using this criterion the bugs were identified as survivors or victims and then they were put in individual randomly placed bottles which were numbered so that each bug had a number as an identity for recognizing it. The length of each bug was recorded with a scale. Then each of these bugs was put in a petri dish containing damped cotton balls and the petri dish was placed under the stereomicroscope to count the number of plates. The weight of each bug was noted down. [4] For recording the speed of the bugs two scales were arranged on a paper adjacently to create an array and the enclosed space between the scales was filled with mud. A starting line was marked, the bug was placed at a certain distance behind the starting line and was gently poked with a paint brush to initiate it to run. The time was noted for covering a distance of $10 \mathrm{~cm}$ from the starting line and using this the speed was calculated. A second run was performed in a similar way after 30 mins and readings were recorded. Both the speeds were used to calculate the average speed 
for each bug. [5] For the next evaluation 5 inches by 10 inches chamber filled with moist mud was used and a mesh was placed in such a way that it divided the chamber into 50 boxes. Now males were placed in even boxes and the females were placed in odd boxes. Once the placement was done the movement of these bugs out of their area in 5 seconds was noted down. Those left their area were recorded as 1 and those who did not be recorded as 0. [6] The mesh was removed and in two corners of the chamber small enclosures of mud were made which had water in it, the other two corners were considered the dry ends. One of each corner with and without water was illuminated with light and the others were considered the darker regions. The bug was dropped in the center and its movement was recorded. The movement towards water was recorded as 1 and towards the drier end was recorded as 0 , similarly, the movement towards dark was recorded as 1 and towards light was recorded as 0. [7] The experiment was performed with five predation tools. For each predation tool, the experiment was performed four times, two times when the bugs were fed on a high-fat diet and the other two times when the bugs were fed on a low-fat diet. For each set of high-fat diet and low-fat diet, one set was for Portuguese origin and the other was of Ukrainian origin. [8] Out of the collected data for each predator now the survivor males and females were identified and separately analyzed to see which range of length, weight and age enabled higher average speed which in turn enables higher survival for them. Similarly, bugs from all trials were selected for each predation tool. Using length as the basis the relation of all these selected bugs with age, weight and average speed was identified. This was the first analysis to select bugs. [9] A second analysis for selection of bugs was done by comparing all the bugs for each predation tool using the parameters of diet and movement towards the water and towards dark, as described in points [5] and [6].The selection was made using the parameter which enabled higher survival in each trial. The relation identified in the first analysis was then implied on the bugs selected using second analysis. The bugs which were closest to the identified relation were the final selected bugs for the generation. [10] The fittest males and females were now identified as the parent generation and were allowed to mate for 7 days and then the first filial generation was obtained.Similarly using the above method the fittest survivors over ten filial generations were identified.[11] A phenogram was made for all the generations by comparing all parameters. The phenogram was used to identify the most recent common ancestors for the bugs and those bugs which showed similarity to these ancestors were identified and removed from the compiled selected bugs over all generations. This was done to identify the bugs which had shown deviation i.e. had evolved.[12] The bugs selected in the last step were now again segregated as males and females. From both of these sets, the ideal bug showing maximum survival was identified by analyzing the parameters which enabled a maximum average speed.The selected male bug was compared to male bugs over all the generations and the relative difference in length, weight, and the average speed was calculated. The bugs showing least relative difference were selected from all the generations. Similarly, all the females were analyzed and bugs were selected over all the generations.These bugs were the final selected bugs.[13] The males and females who were finally selected were mapped continuously over a hundred seed values using mesquite.The bugs showing maximum deviation were identified and their frequency was noted. A graph was then plotted between the continuously mapped generations and the hundred tests performed to understand the trend and microevolutionary forces involved.Further, a computer model was used to understand and verify the microevolutionary forces involved.[14] DNA fingerprinting of selected bugs from each generation and category(male and female) was done, to 
determine their origin. Stipulated data was verified from computer model generated by Gene Compiler.[15] Bovine serum assay test of finally selected bugs was done in order to set the relation between protein concentration and micro-evolution.

\section{[16] Protocols used are as follows;}

DNA Isolation: [1]. Dried alcohol dipped samples were transferred microcentrifuge tubes. [2]. 333k prewarmed CTAB (600ul) +3ul of Beta-Mercaptoethanol $10 u l$ of $20 \%$ SDS was added. [3]. After crushing 3ul of Pronase was added in each tube. [4]. each tube was vortexed for 5 minutes vigorously.[5]. Samples were Kept overnight for incubation, and vortexed after the interval of 2-3 hours if possible, after incubation, samples were brought to room temperature and centrifuged at 14,000 $\mathrm{rpm}$ for $10 \mathrm{~min}$. [6]. an equal volume of Phenol: Chloroform: Isoamyl alcohol (25:24:1) was added and mixed for 5 minutes (vortex).[7]. After mixing, centrifuged at 14,000 rpm for 10 minutes.[8]. Then, the supernatant was collected in a fresh microfuge tube and the pellet was discarded.[9].Chloroform: isoamyl alcohol (24:1), 600ul in each tube was added and mixed(vortex).[10]. Centrifuge at $12,000 \mathrm{rpm}$ for 10 minutes.[11]. Take the supernatant in a fresh microfuge tube and discard the debris.[12.] Add chilled isopropanol (400 ul) and mixed slowly until white flakes appear.[13]. The supernatant was poured and add $70 \%$ chilled ethanol (400ul) +ammonium acetate (100 ul) to the pellet was added for washing.[14]. Centrifuged at 10,000 rpm for 10 minutes.[15]. the supernatant was poured carefully; 400ul of absolute alcohol was added and Centrifuged at $10,000 \mathrm{rpm}$ for 10 minutes.[16]. Decant the supernatant and dry the pellet at room temperature.[16]. Dissolve it in nuclease-free water.

Protein Isolation: [1]. Dried alcohol dipped samples were transferred microcentrifuge tubes. [2]. 333k prewarmed CTAB (600ul) +3ul of Beta-Mercaptoethanol $10 u l$ of $20 \%$ SDS was added. [3]. After crushing 3ul of
DNAse and RNAse was added in each tube. [4]. each tube was vortexed for 5 minutes vigorously.[5]. Samples were Kept overnight for incubation, and vortexed after the interval of 2-3 hours if possible, after incubation, samples were brought to room temperature and centrifuged at $14,000 \mathrm{rpm}$ for $10 \mathrm{~min}$. [6]. an equal volume of Phenol: Chloroform: Isoamyl alcohol (25:24:1) was added and mixed for 5 minutes (vortex).[7]. After mixing, centrifuged at 14,000 rpm for 10 minutes.[8]. Then, the supernatant was collected in a fresh microfuge tube and the pellet was discarded.[9].Chloroform: isoamyl alcohol (24:1), $600 \mathrm{ul}$ in each tube was added and mixed(vortex).[10]. Centrifuge at 12,000 rpm for 10 minutes.[11]. Take the supernatant in a fresh microfuge tube and discard the debris.[12.] Add chilled isopropanol (400 ul) and mixed slowly until white flakes appear.[13]. The supernatant was poured and add $70 \%$ chilled ethanol (400ul) +ammonium acetate (100 ul) to the pellet was added for washing.[14]. Centrifuged at 10,000 rpm for 10 minutes.[15]. the supernatant was poured carefully; 400ul of absolute alcohol was added and Centrifuged at $10,000 \mathrm{rpm}$ for 10 minutes.[16]. Decant the supernatant and dry the pellet at room temperature.[16]. Dissolve it in nuclease-free water.

\section{CTAB EXTRACTION BUFFER - (For 50ml)} $1 \mathrm{M}$ Tris- $\mathrm{HCl}$ 5ml,0.5 M EDTA 2ml,5M NaCl $17.5 \mathrm{ml}$ $10 \%$ CTAB $10 \mathrm{ml}$ (5 gm in $50 \mathrm{ml})$, Distilled Water 15.5 $\mathrm{ml}$ to make up the volume.

Bicinchoninic Acid Protein Assay:[1]. Pipette 25 $\mu \mathrm{L}$ of each standard or unknown sample replicate into a microplate well (working range $=20-2000 \mu \mathrm{g} / \mathrm{mL}$ ) [2] Add $200 \mu \mathrm{L}$ of the WR to each well and mix plate thoroughly on a plate shaker for 30 seconds. [3]. Cover plate and incubate at $37^{\circ} \mathrm{C}$ for 30 minutes. [4]. Cool plate to RT. Measure the absorbance at or near $562 \mathrm{~nm}$ on a plate reader. [5].Subtract the average $562 \mathrm{~nm}$ absorbance measurement of the Blank standard replicates from the 562nm measurements of 
all other individual standard and unknown sample replicates. [6]. Prepare a standard curve by plotting the average Blank-corrected 562nm measurement for each BSA standard vs. its concentration in $\mu \mathrm{g} / \mathrm{mL}$. Use the standard curve to determine the protein concentration of each unknown sample.Note:-The test was performed in set of doublets

[17] Further, using, a formula from Punnett square for linking natural selection with genetic drift and further adaptive radiation. Considering Gene belonging to the origin Ukraine (U) as dominant and Gene belonging to the origin Portugal $(\mathrm{u})$ as recessive, Parental Generation was plotted between UU and uu, For all the subsequent generations the Punnett square(s) were made by choosing the genes (as origins) of males and females which was evident from the results obtained from adaptive radiation. The results obtained from all the squares were used to determine the ratio between bugs belonging to origin Ukraine to that of belonging to the origin of Portugal. The ratio was 23:21. This ratio was compared to a generalized ratio obtained from Punnett square and equating the ratio using constants "a" and " $b$ " the value for "a" and "b" were determined. The value obtained from dividing constants "a" and "b" was found out to be - 2.7432122 which was kept as a multiplying factor for the derived equation. [18] Now the character(s)- length and weight were analyzed. Considering gene expressed as a heavyweight (W) and gene expressing short height as dominant (L). The bugs belonging to origin Ukraine were WWLL and that to Portugal were wwll. The parental cross was made between WWLL* wwll, resulting in filial one generation to be $\mathrm{WwLl}$. Then crossing of filial one generation i.e. $\mathrm{WwLl} * \mathrm{WwLl}$ the results were analyzed to obtain the percentage of bugs showing the phenotype of heavyweight and short height, which was the selected phenotype of selected bugs, selected using simulated natural selection. The required percentage was $56.25 \%$. Now the percentage was multiplied to the ratio $23: 21$ derived initially, to determine $\mathrm{z}_{1}$ and $\mathrm{z}_{2}$ which were the required frequencies obtained from the observed results. Multiplying $\mathrm{z}_{1}$ and $\mathrm{z}_{2}$ with a total number of observed population i.e. 100 , the values of $\mathrm{p}^{2}$ and $\mathrm{q}^{2}$ were obtained respectively. Using Hardy Weinberg principle the equation was simplified by putting the values of $\mathrm{p}^{2}$ and $\mathrm{q}^{2}$ and the value for $2 \mathrm{pq}$ was determined. Further, substituting the values for $\mathrm{p}$ and $\mathrm{q}$ in $(\mathrm{p}+\mathrm{q})^{2}$ resulting 112.08 which is greater than 100 , hence signifying evolution. The same calculation can also be done without multiplying the result with hundred then the result would be in terms of frequency rather than a number. All the obtained results were substituted in a derived mathematical equation and finally, the mathematical equation linking natural selection and genetic drift using adaptive radiation was derived and verified.

\section{Observation}

The selection of bugs over 10 filial generations was done considering various characters namely predation, length, number of plates, age, weight, average speed, movement out of an allotted area in 5 seconds, the movement towards dark and movement towards the water. The average speed of all the selected bugs has been summarized in Figure 1. Studying the data for the three characters length, weight and average speed individually (Figure 2), it was observed that average speed showed maximum deviation in terms of the spread of data and this made it evident that was the defining character for the selection of bugs. For further screening, the process of selecting the effect of other characters in terms of average speed was compared. Figure 3 and Figure 4 represent the plot of mean values for different characters with average speed and then a comparison of effect in a Pareto. It was evident that the characters weight and length with a standard deviation greater than 2 had a greater impact in comparison to other characters like age and 
number of plates. Hence the characters length and weight in comparison to average weight were used for the selection of bugs.

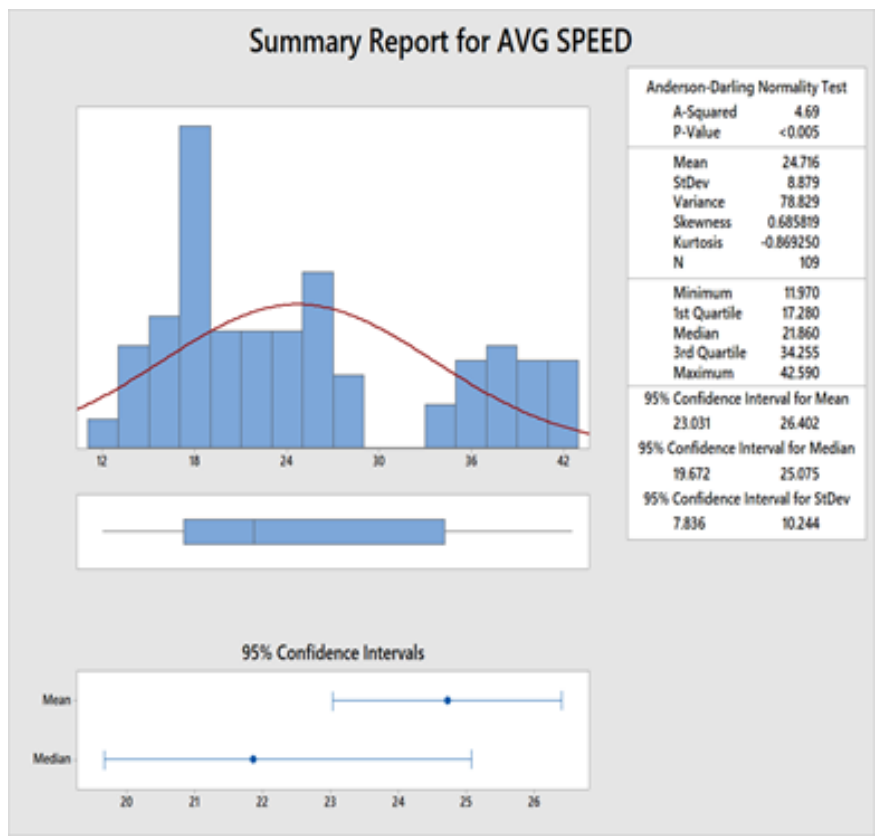

Figure 1

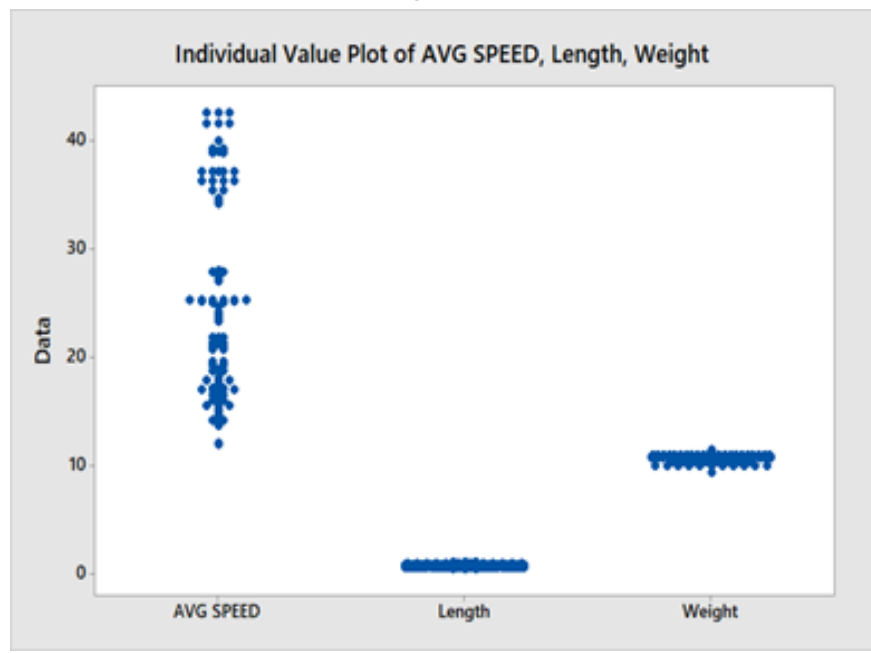

Figure 2

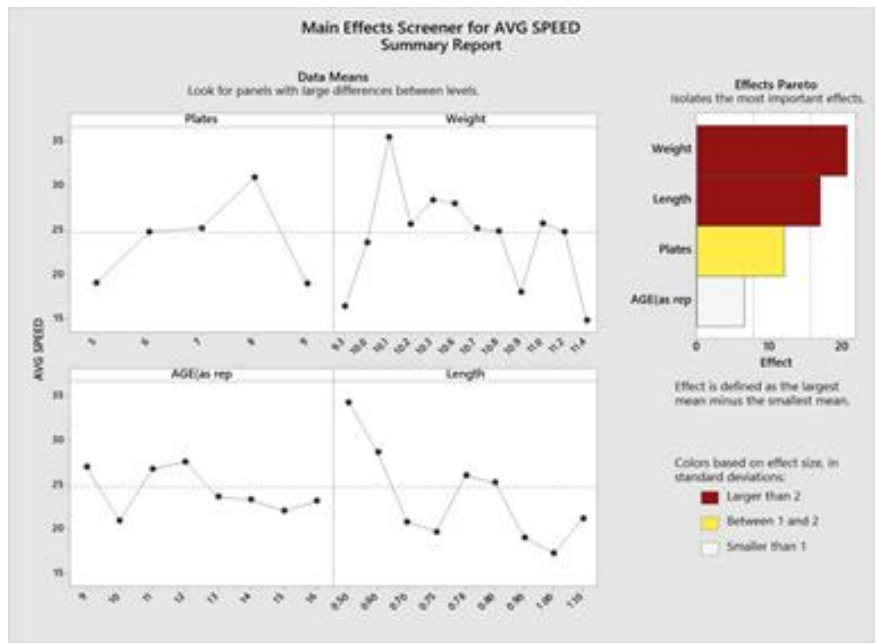

Figure 3

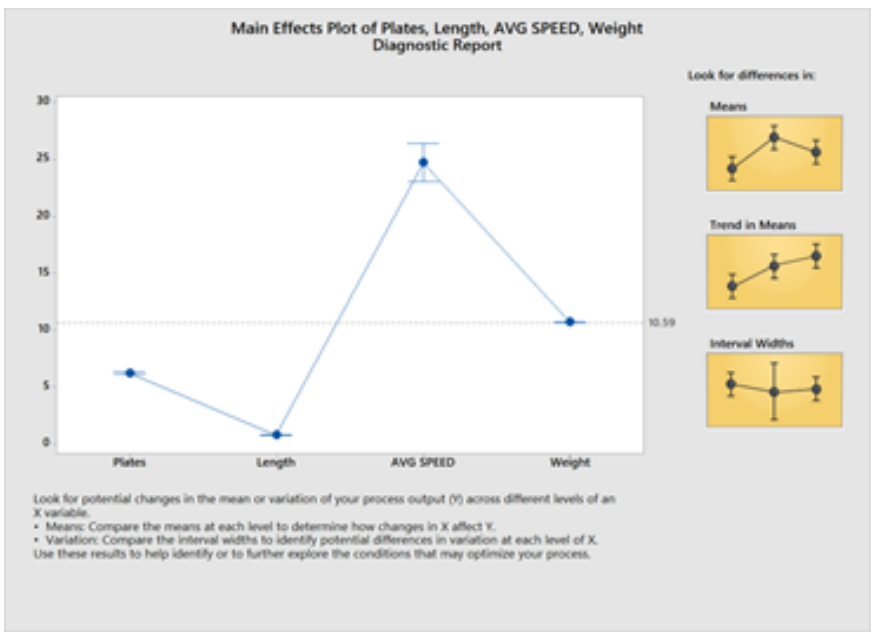

Figure 4

Analyzing the length for bugs, shorter bugs enabled higher survival. The length of the selected bugs is summarised in Figure 5. Further, an I-MR Chart i.e. an Individual-Moving Range chart was plotted to check for the stability of data so that further capability analysis could be performed. Figure 6 shows the I-MR Chart which shows that the data was stable. Further, capability analysis was performed on the upper limit 1 and lower limit 0.6. The $\mathrm{z}$ potential value for the range was 1.48 which indicated moderately high capability. Figure 7 shows the capability analysis in terms of actual and potential values whereas Figure 8 represents the $\mathrm{z}$ potential for the selected range of length. 


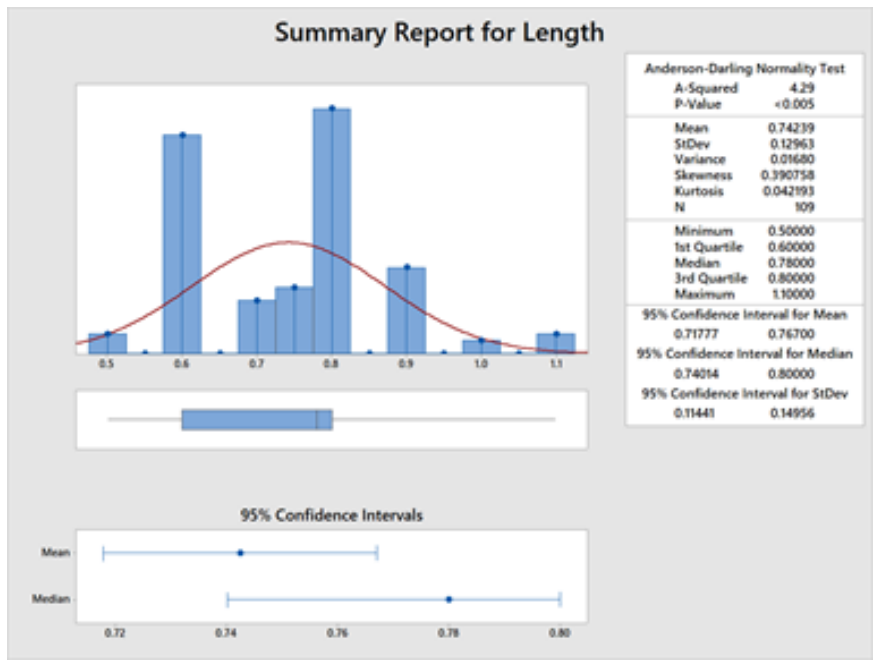

Figure 5

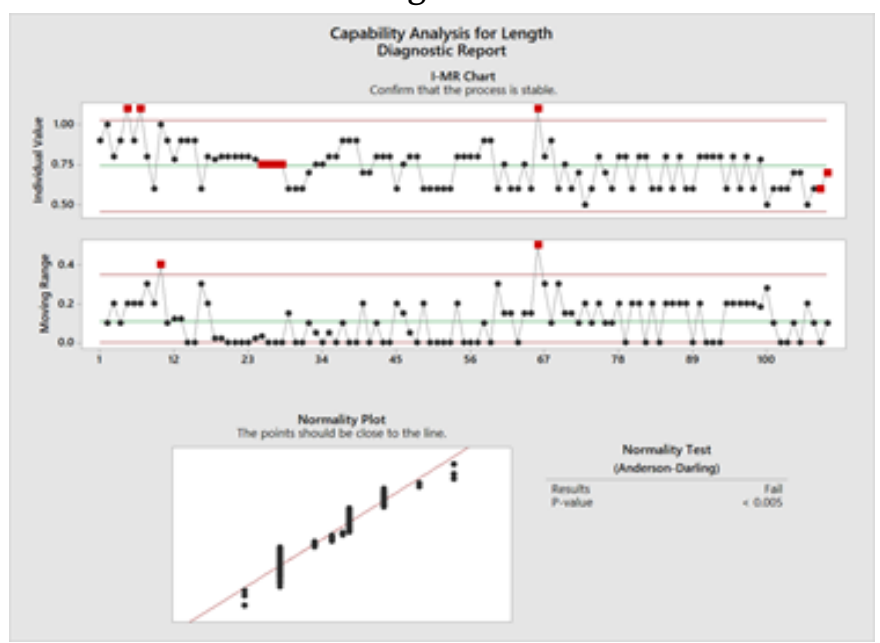

Figure 6

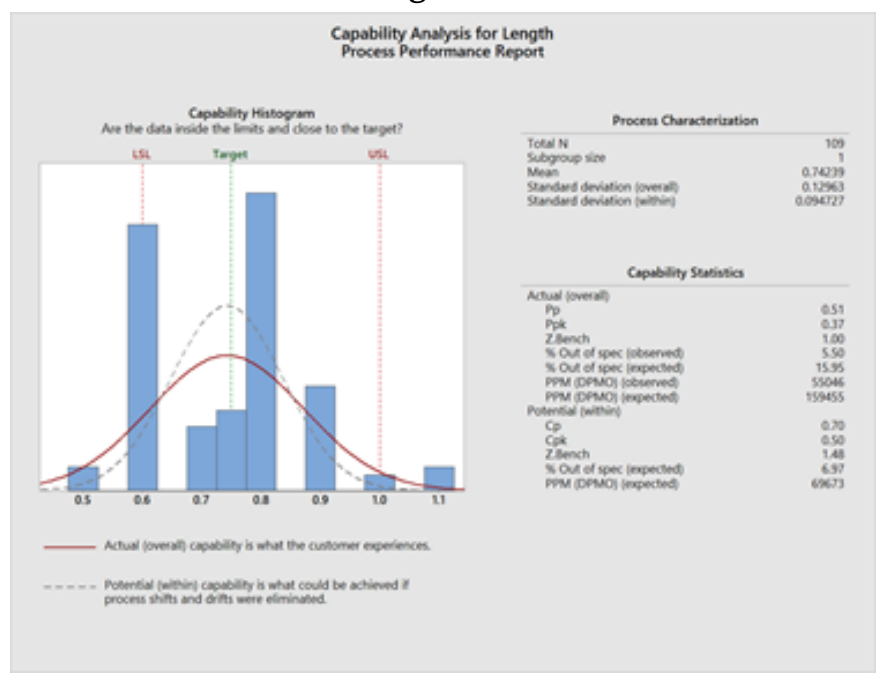

Figure 7

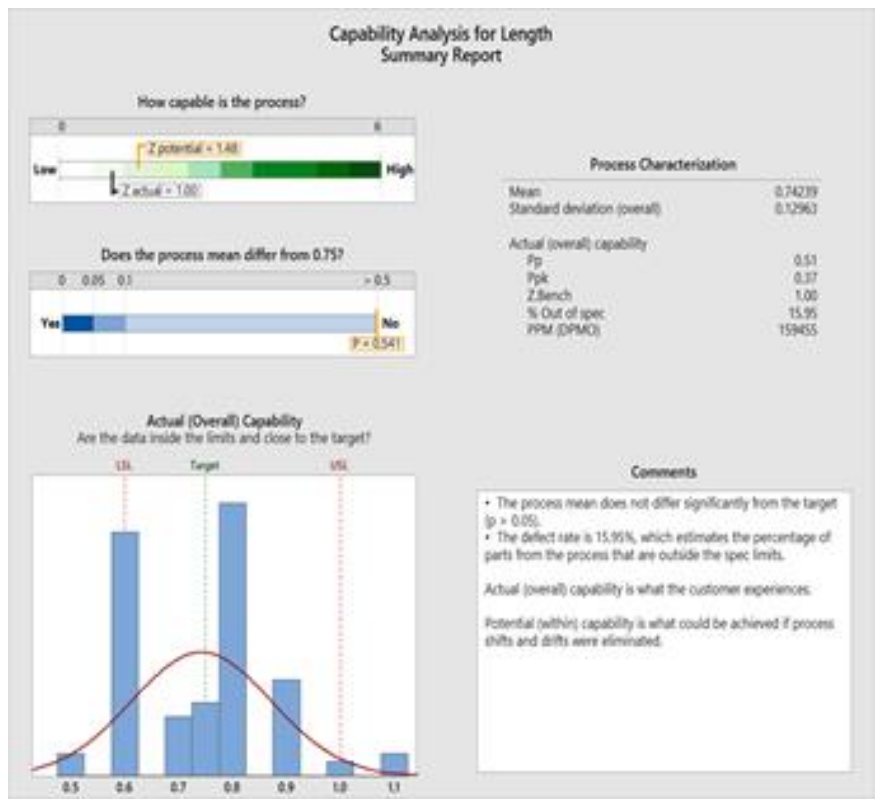

Figure 8

Similarly analyzing weight of the bugs it was evident that moderately heavy bugs had the highest survival. Figure 9 summarizes the weight of all the selected bugs over all the generations. On plotting an I-MR Chart for the weight of bugs Figure 10 it was observed that the data was stable and was fit for further capability analysis. Using 11.4 as the upper limit and 10 as the lower limit the capability analysis was performed. Figure 11 represents the graph for the actual and potential values. Figure 12 represents the $\mathrm{z}$ potential for the weight in the defined in the given range which was 2 that clearly indicates moderately high capability for the process.

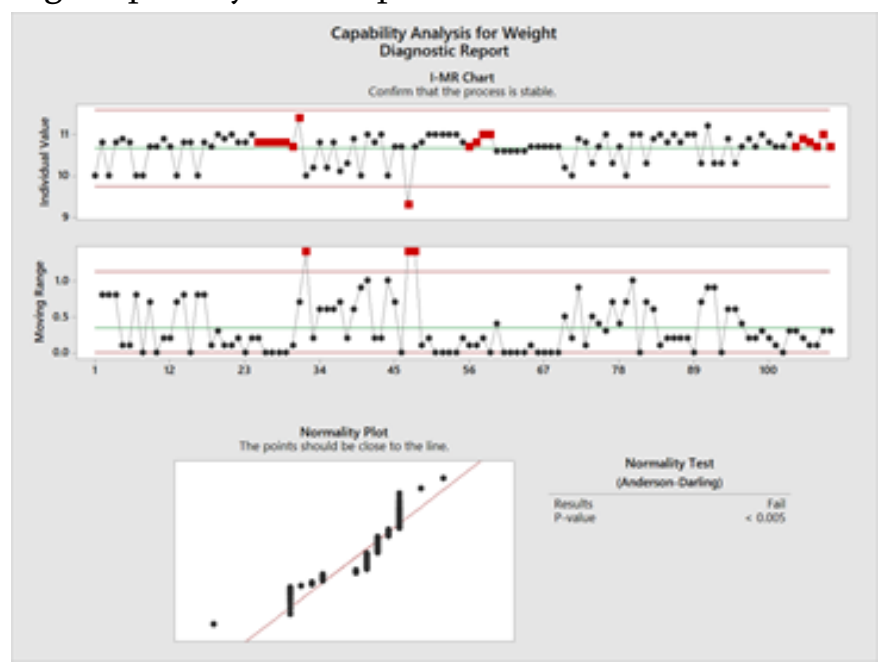

Figure 9 


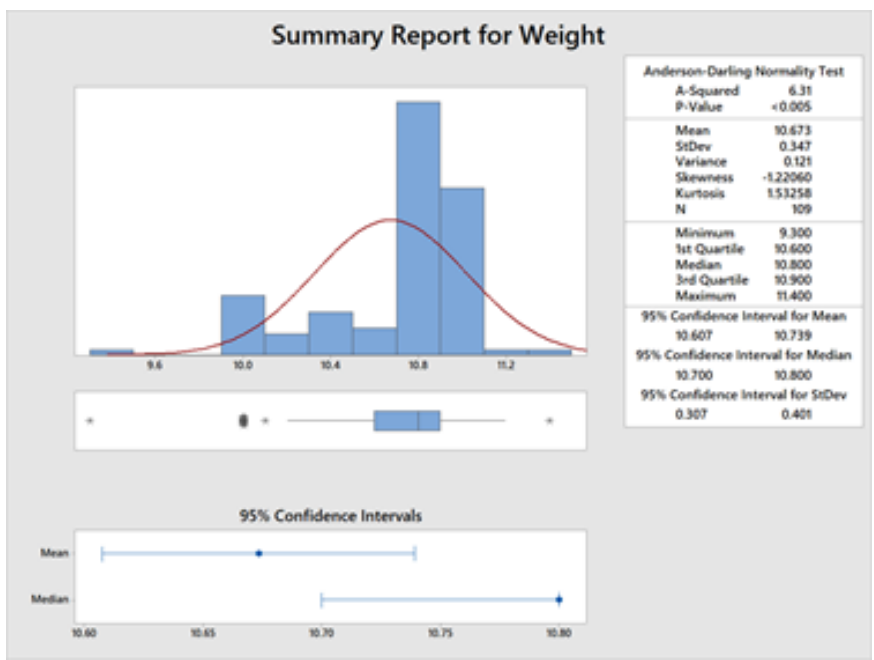

Figure 10

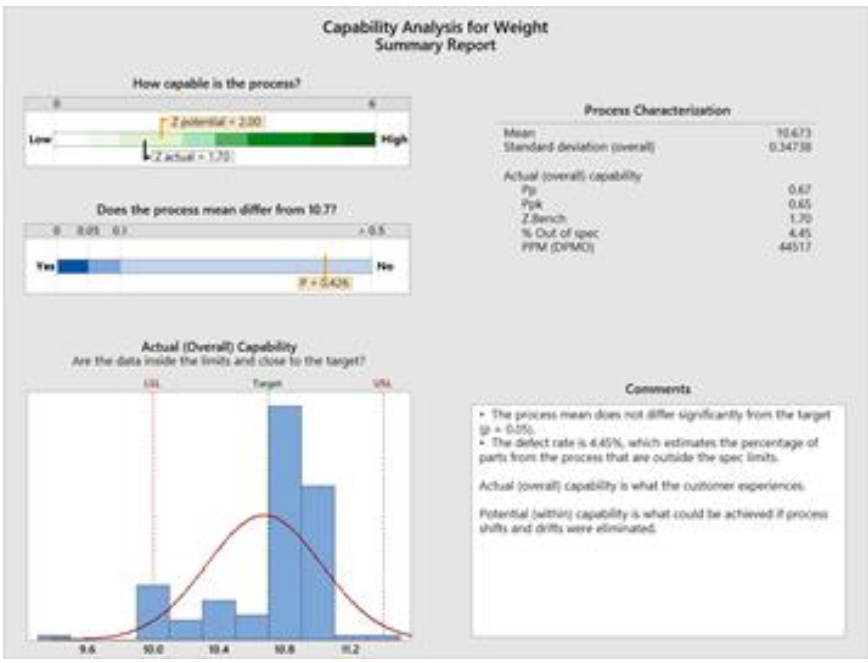

Figure 11

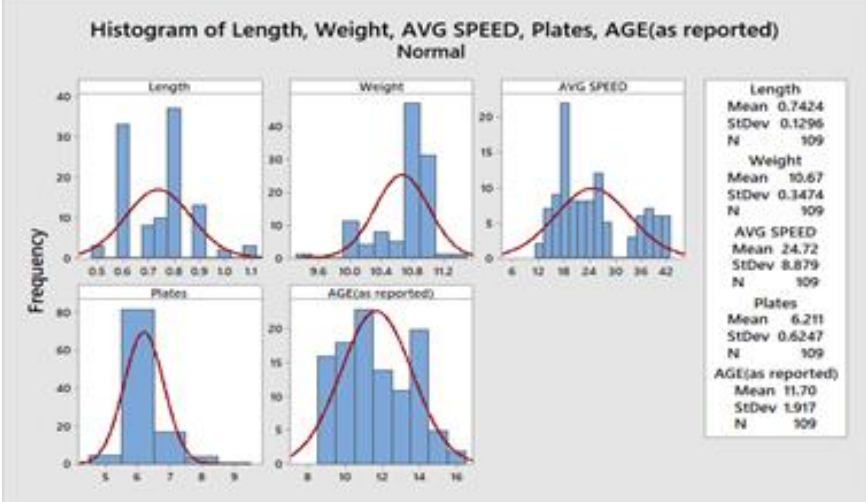

Figure 12

The characters short length and moderately high weight were used for the selection of bugs as these characters enabled higher average speed thereby increasing the survival of the bugs. The capability analysis of length and weight in these ranges had high $\mathrm{z}$ potential which indicated that it was a capable process. The same result can be verified by the contour plot plotted in Figure 13.

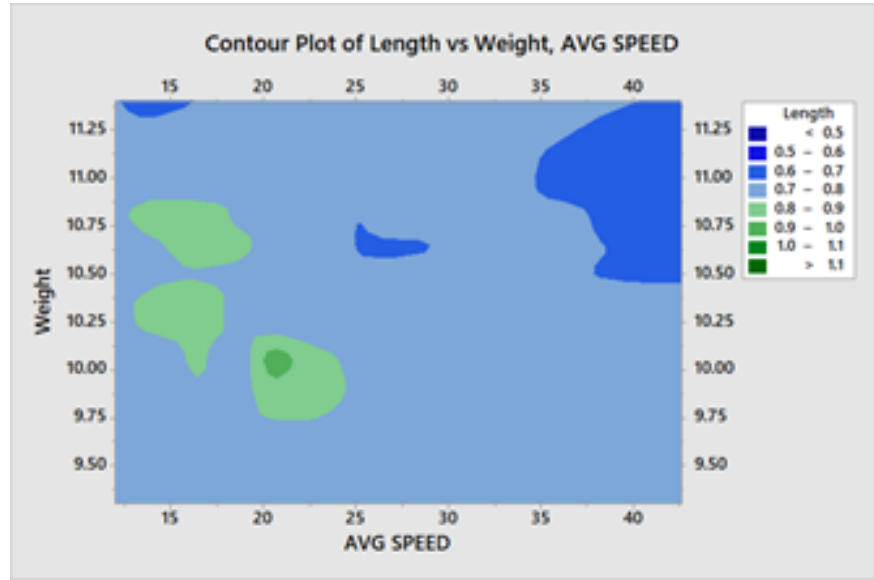

Figure 13

Figure 14 represents the histogram of length, weight, average speed, number of plates and age of selected bugs. For analyzing the bugs over all generations for the mean, range etc. of the above characters boxplots were used. Figure 15 represents the boxplots of bugs for all the generations. The mean length was $0.74 \mathrm{~cm}$, mean weight was $10.6 \mathrm{~g}$ whereas the mean age for the bugs was 11.4

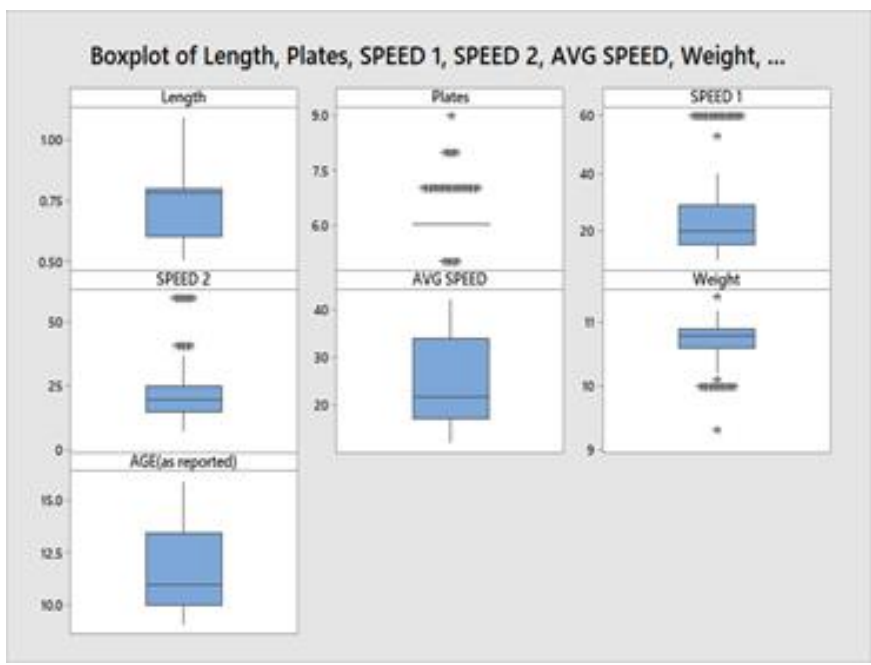

Figure 14 and Figure 15

On performing multiple regression analysis of average speed with length and weight Figure 16 with a goal to maximize average speed, the optimal length according to the model was $0.5 \mathrm{~cm}$ which once again verifies the 
selection of bugs with short length. The prediction plot of the model evidently shows that the data lies in 95\% Prediction interval with the predicted value for average speed as 30.3956 .

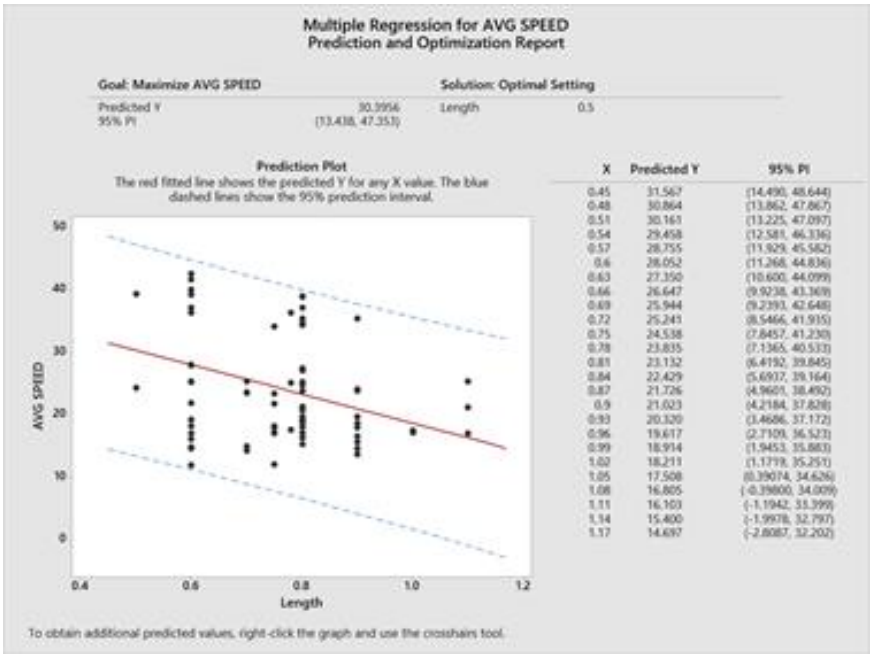

Figure 16

The relation between $\mathrm{X}$ and $\mathrm{Y}$ in the model i.e. average speed with length has a $\mathrm{p}$-value less than 0.001 hence the relationship is statistically significant. Since the regression model fits the data well the regression equations formed were later used to equate and derive a mathematical formula. The graphs of average speed with Length and Weight as $\mathrm{X}$ variables can be seen in Figure 17. Moreover, on plotting residuals versus fitted values Figure 18 an unequal variation is observed which is because natural selection has been simulated randomly over 1000 bugs.

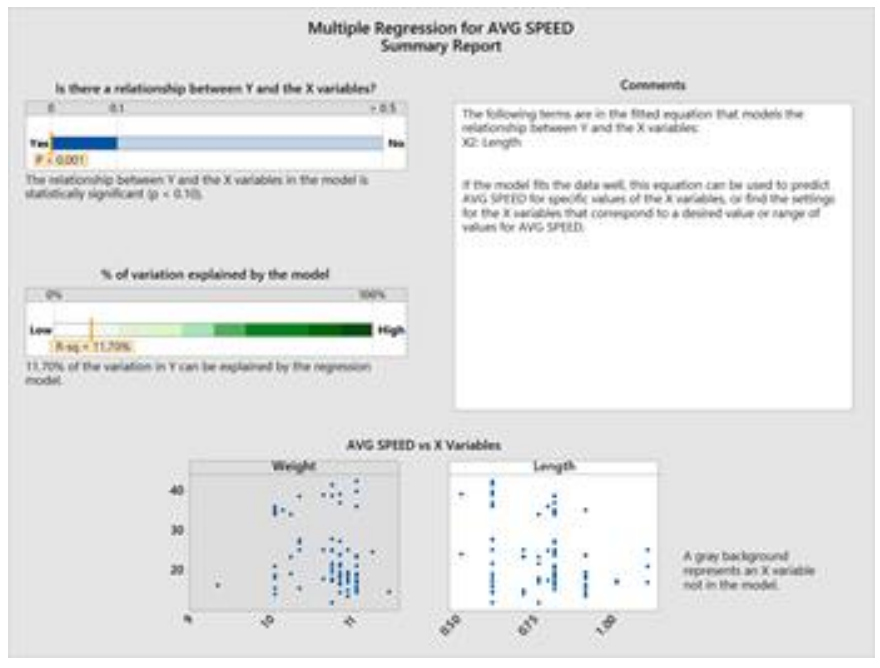

Figure 17

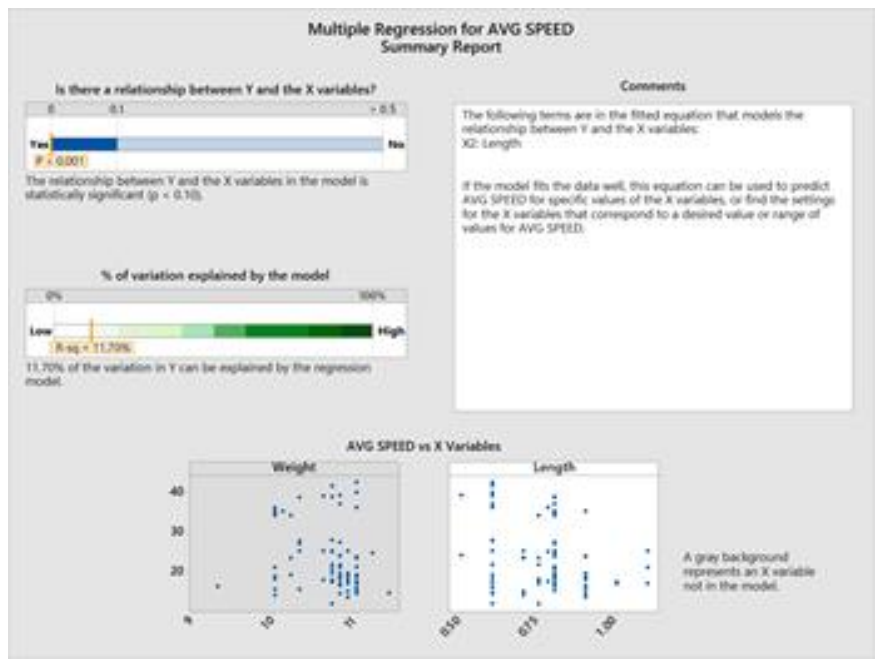

Figure 18 and Figure 19

Analyzing the interaction plot between average speed, length and weight Figure 19 the observed pattern clearly indicates the presence of interaction between average speed which is a physical environmental factor with the factors length and weight at the genetic level. This interaction establishes a geneenvironment interaction. The same result is obtained by reversing length and weight which is evident in Figure 20.

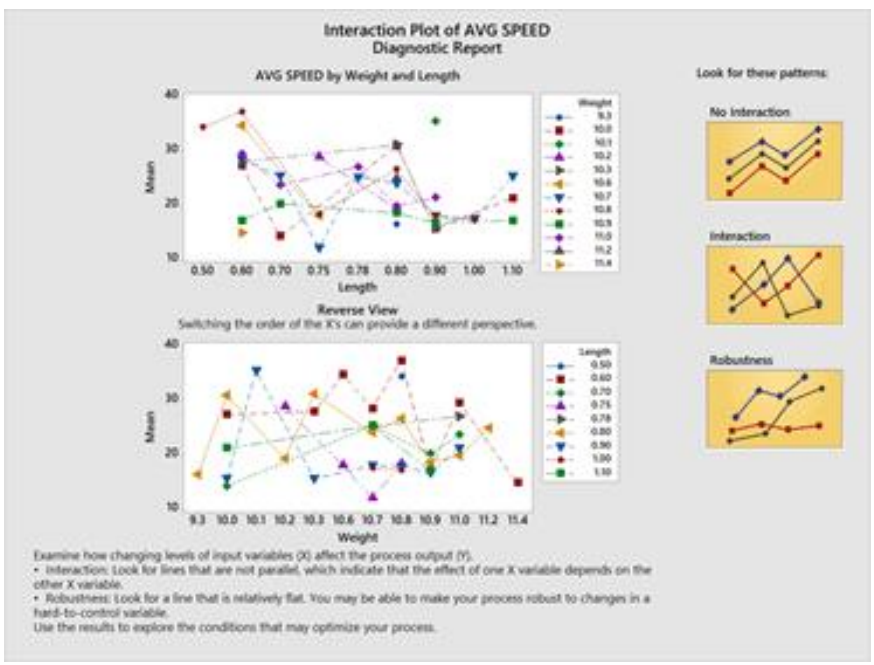

Figure 20

Further, a matrix plot was plotted between the characters length, weight, average speed, and age. Matrix plot 1 is a matrix plot between these 
characters for the bugs for all the generations i.e. from the parent generation to the tenth generation. Matrix plot 2 has been similarly plotted for the selected survivors chosen from all the bugs. Analyzing both the matrix plots it becomes evident that the character length exhibits a major deviation in the survivors in comparison to other characters. Maximum deviation in length again throws light on length becoming a defining character for selection, verifying and supporting the previous observations.

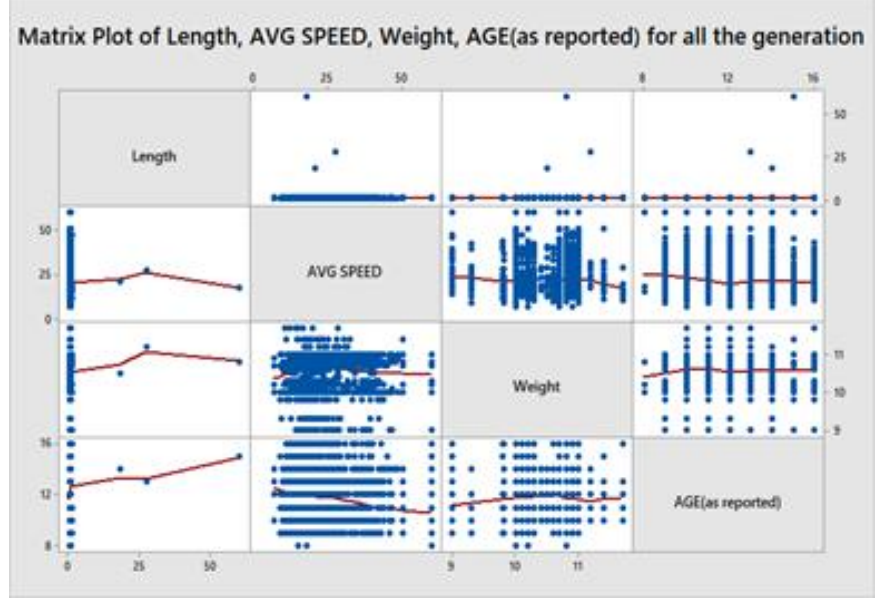

Matrix Plot 1

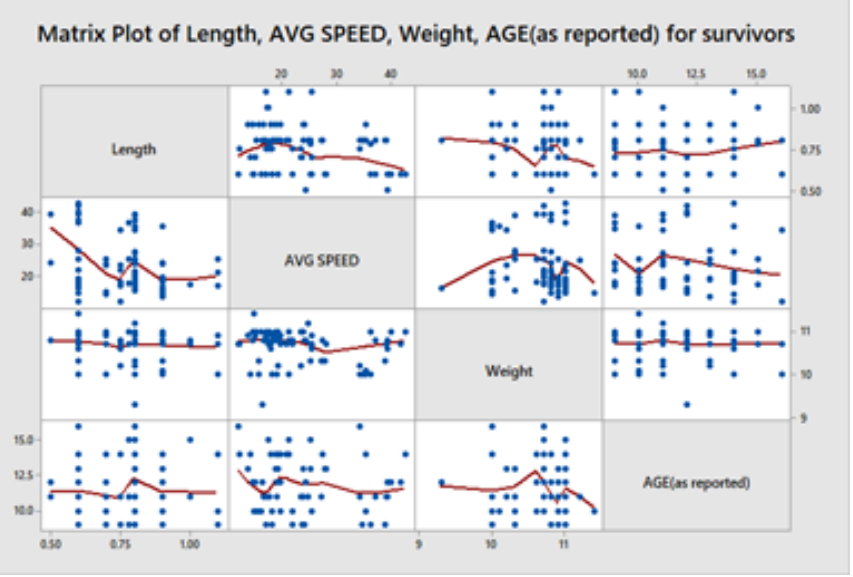

Matrix Plot 2

The bugs were fed with high fat and low fat diets. On comparing the selected bugs it became evident that more bugs with high fat diet were selected. This result can be understood with Figure 21 and Figure 22. Figure 21 shows the comparative histogram of speed 1 , speed 2 and average speed recorded for the bugs fed with high fat diet. It is evident that speed 1 for the bugs is lower than speed 2 for most of the bugs. This is because when the bugs were fed on high fat diet they had higher energy stored, which enabled them to run faster in the second run. The stored energy thereby enables better survival and it is evident that the average speed is more symmetric for the data which further justifies the selection of more bugs with high fat diet. Figure 22 shows speed 1, speed 2 and average speed for bugs fed with low fat diet. It is evident from the graph that speed 1 and speed 2 for graphs almost overlaps for majority of the bugs. This is because the bugs fed with low fat diet have less energy for the second run as a result they become more vulnerable to predation.

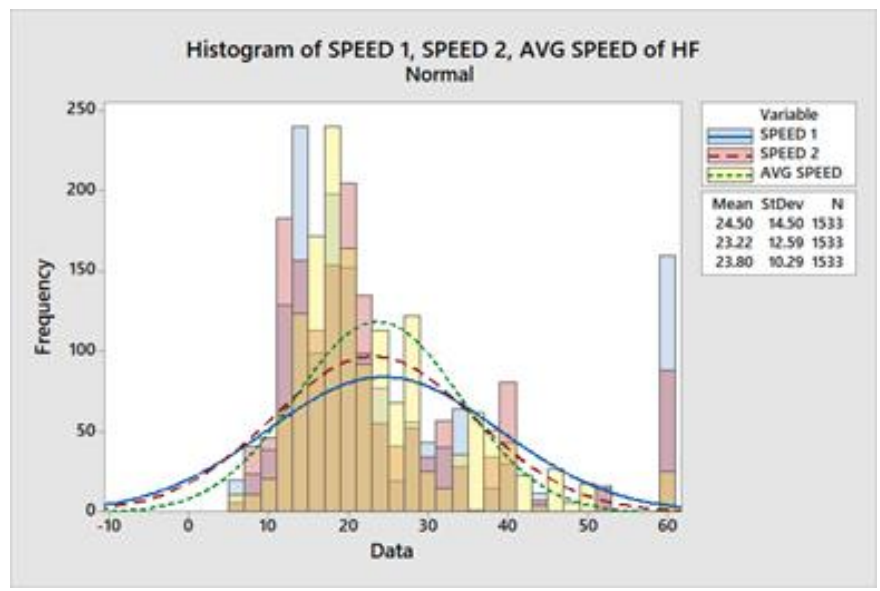

Figure 21

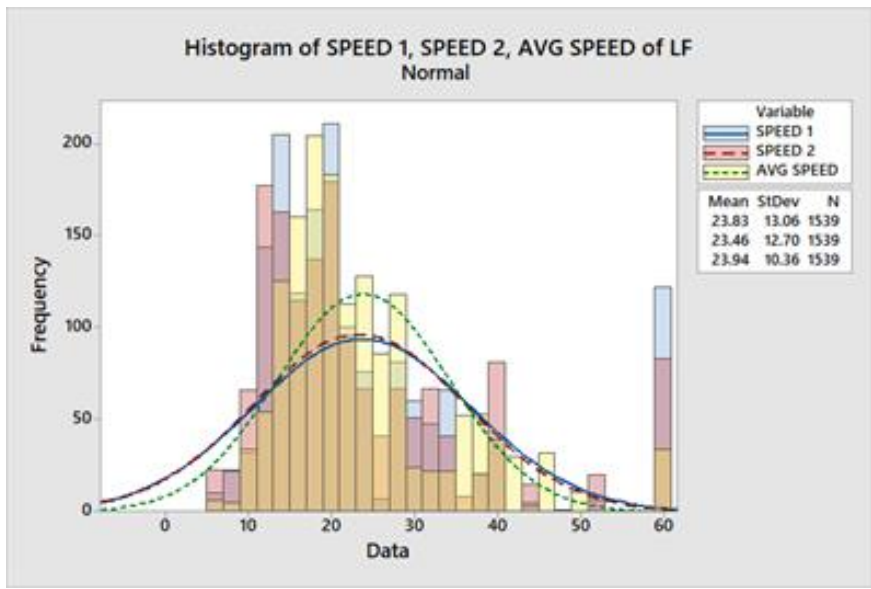

Figure 22

Therefore summing up all the observations, short length, moderately heavy weight and high fat diet were the favourable characters which enabled higher average speed for bugs thereby increasing their 
survival. Further, a phenogram Figure 23 was made for the bugs selected from all the generations using the above defined parameters. The phenogram was studied to find out the most recent common ancestors.Consider the Figure 23.1, different nodes pointing out to common ancestry have been named. The 4th node points out to the most common recent ancestors for the second and tenth generation. Similarly, other nodes point out to the most common recent ancestors.Finally the 1 st node points out to parent generation. Now considering the fifth and eighth generation. The bugs were analyzed to see the characters from which generation were dominantly present. It was observed that average speed differed for both generations and more bugs with average speed from the eighth generation were found out. The bugs similar showing characters similar to these were identified and these bugs were the bugs showing traits from the most recent ancestor defined by the 5 th node. Similarly using this method the bugs showing similarity with the 4 th and 6 th node were identified. The same method was implied on the selected bugs to further find out the 2nd and 3rd node.

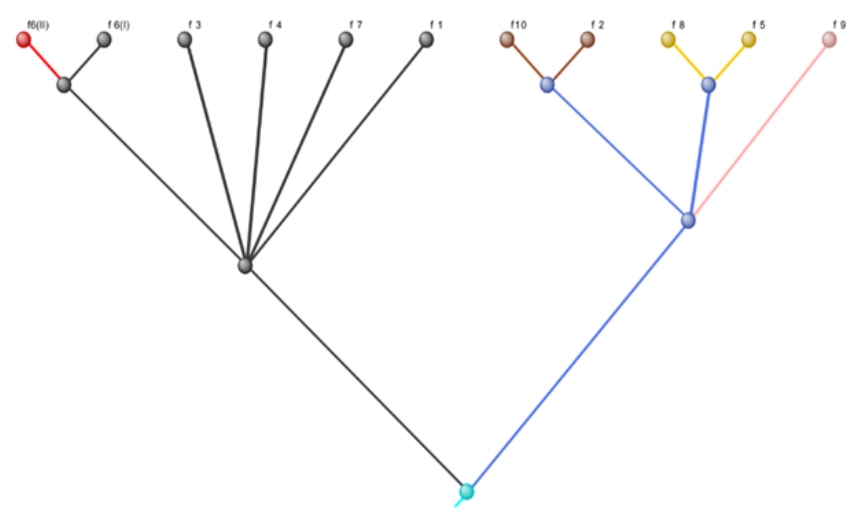

Figure 23

Once these bugs showing traits similar to common ancestors were identified they were removed from the present generations as these were the bugs which had not gone evolution. From all the generations now only those bugs were present which had some difference in traits as compared to common ancestors i.e only bugs showing evolution were now studied. These selected bugs were segregated into males and females. Once again analyzing the characters length and weight for maximum average speed an ideal male bug with length $0.8 \mathrm{~cm}$, weight $10.8 \mathrm{~g}$ and average speed 37.19 was selected. Similarly, an ideal female with length $0.6 \mathrm{~cm}$, weight $10.7 \mathrm{~g}$ and average speed 25.3 was selected. The length, weight and average speed of the ideal male and female were compared with the males and females over all generations to find out the relative difference for these parameters. From each generation the bugs showing least relative difference were identified and finally the set of bugs obtained from all the generations showed no difference in length. Figure 24 and Figure 25 show the relative difference of weight observed for males and females respectively whereas Figure 26 and Figure 27 show the relative difference of average speed for males and females respectively.

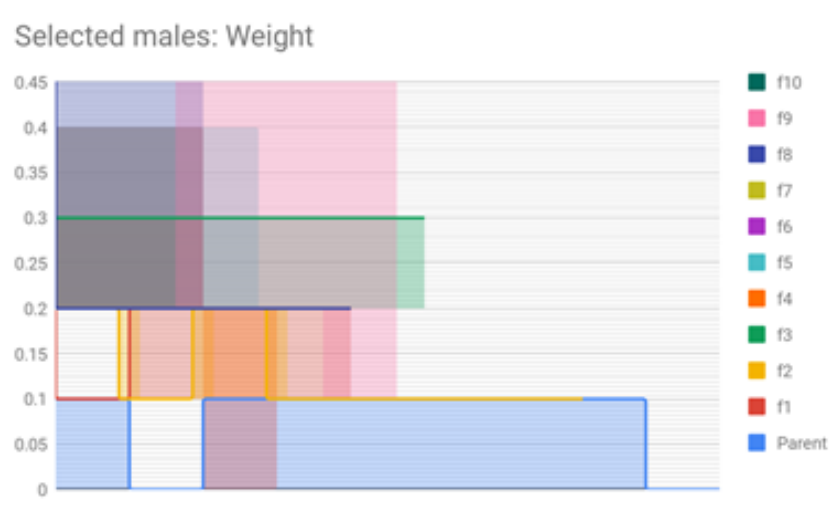

Figure 24

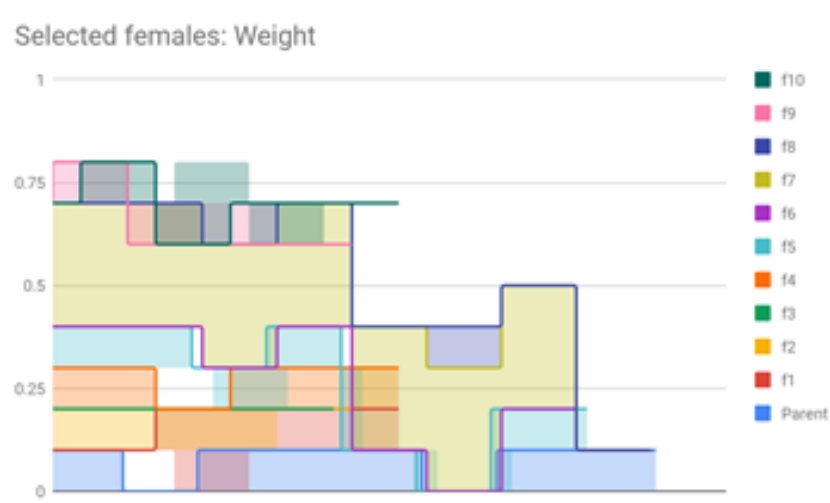

Figure 25 
Selected males: Average speed

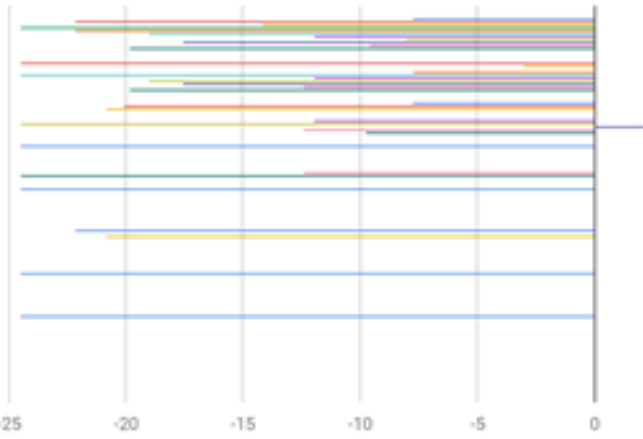

Figure 26

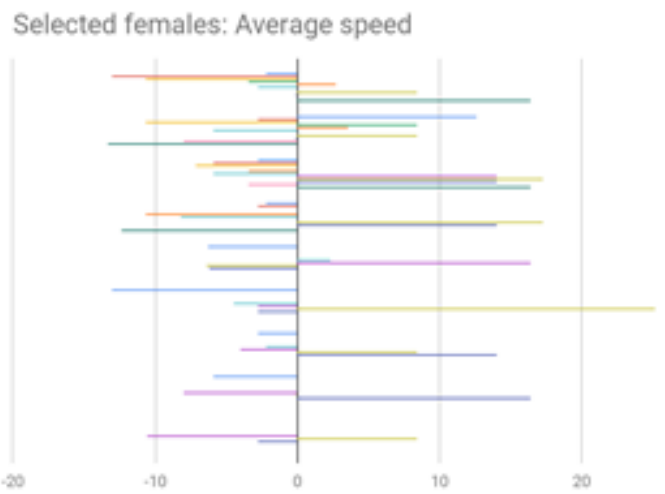

Figure 27
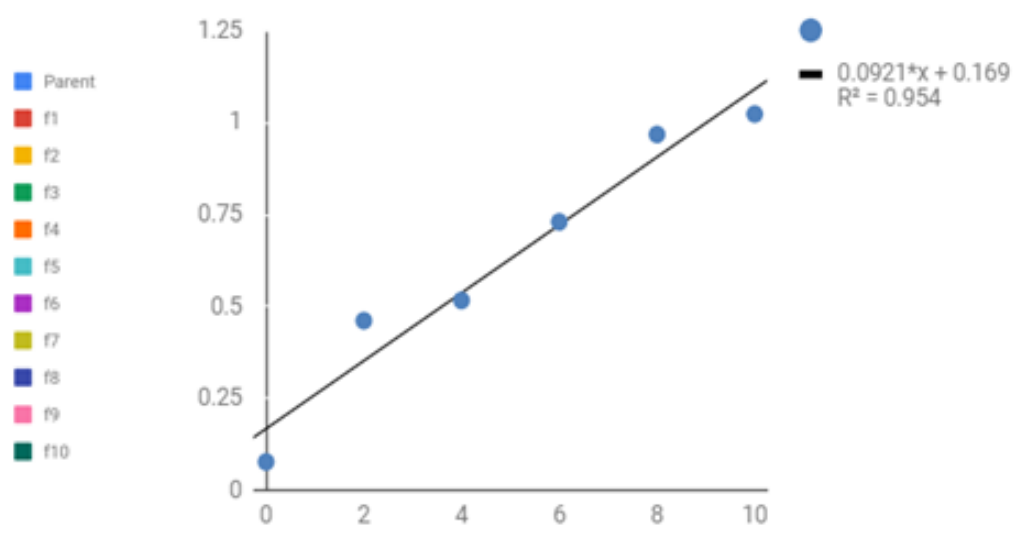

Figure 29

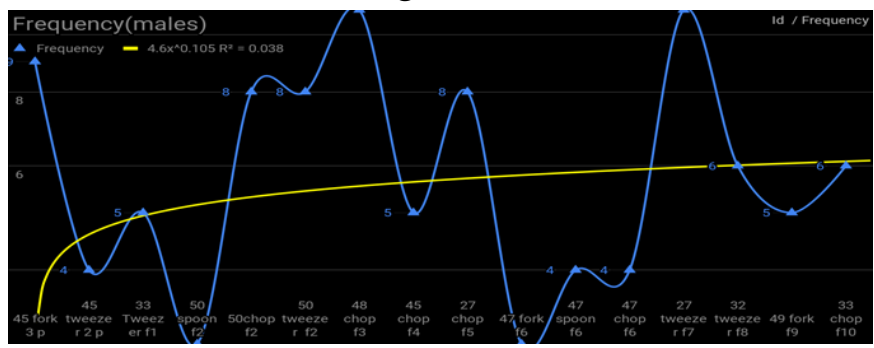

Figure 30

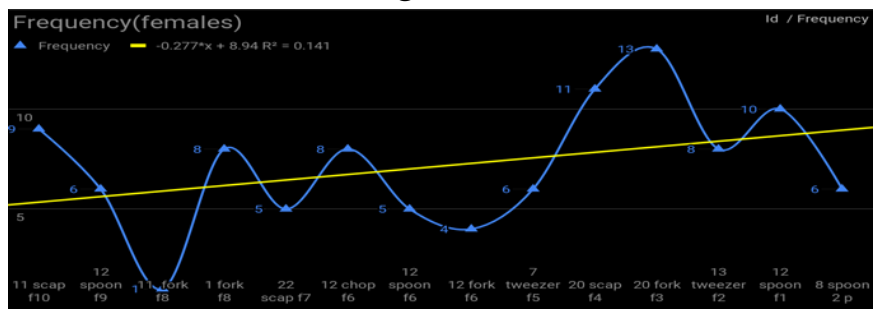

Figure 31

The generation of the bugs showing maximum deviation in each test was noted and the frequency of different generations over these hundred tests for males and females was found out. Figure 30 and Figure 31 shows the frequency of occurrence of the bugs from different generation in the hundred test for males and females respectively.

Then to determine the concentration of proteins in the selected bugs Bovine Serum Albumin Assay test was performed. The test was performed by absorbance of wavelength of $562 \mathrm{~nm}$ with bandwidth equal to $9 \mathrm{~nm}$ and number of flashes equal to 25 . The settle time was $0 \mathrm{~ms}$ and the part of plate used was A4-D5; A1-F2. The temperature was maintained as 293.75 K throughout the experiment. The convention 
used for the experiment for different bugs is given in Table 1.

Table 1.

\begin{tabular}{|l|r|l|}
\hline a & 48 chop f3 & MALE \\
\hline b & 27 tweezer f7 & MALE \\
\hline c & 20 fork f3 & FEMALE \\
\hline d & 20 scap f4 & FEMALE \\
\hline e & 45 fork 3 p & MALE \\
\hline $\mathrm{f}$ & 12 spoon $\mathrm{f} 1$ & FEMALE \\
\hline
\end{tabular}

The test was performed for samples in doublets. Table 2 shows the readings for the test performed in doublets at dilution 10. Further, the BSA plot (Figure 32) was used to calculate $2 X$. The equation obtained was $0.0921 \mathrm{x}+0.169 ; \mathrm{Ax}+\mathrm{b}=\mathrm{y}$, where $2 \mathrm{X}=($ Avg-b)/A. The value for $\mathrm{X}$ was obtained by division by 2 . Finally, the concentration of protein was obtained by dividing 10 i.e. dilution by $1 X$. The negative signs of the concentration are because the concentration is calculated relative to the concentration of BSA therefore a negative deviation gives negative value of concentration.

\begin{tabular}{|r|r|r|r|r|}
\hline Part of Plate & \multicolumn{1}{|c}{1} & 2 & 4 & 5 \\
\hline 3 & 0.07800000161 & 0.07999999821 & 0.174999997 & 0.2389999926 \\
\hline $\mathbf{B}$ & 0.3680999875 & 0.5562000275 & 0.1536000073 & 0.1363999993 \\
\hline 0.5171999931 & 0.5170999765 & 0.1638000011 & 0.145600006 \\
\hline 0.7542999983 & 0.7059999704 & 0.241899997 & 0.2345000058 \\
\hline & 1.021100044 & 0.9136000276 & NA & NA \\
\hline & 0.9894999862 & 1.056100011 & NA & NA \\
\hline DUPLET BSA (DOUBLET) & SAMPLES (DOUBLET)
\end{tabular}

Figure 32

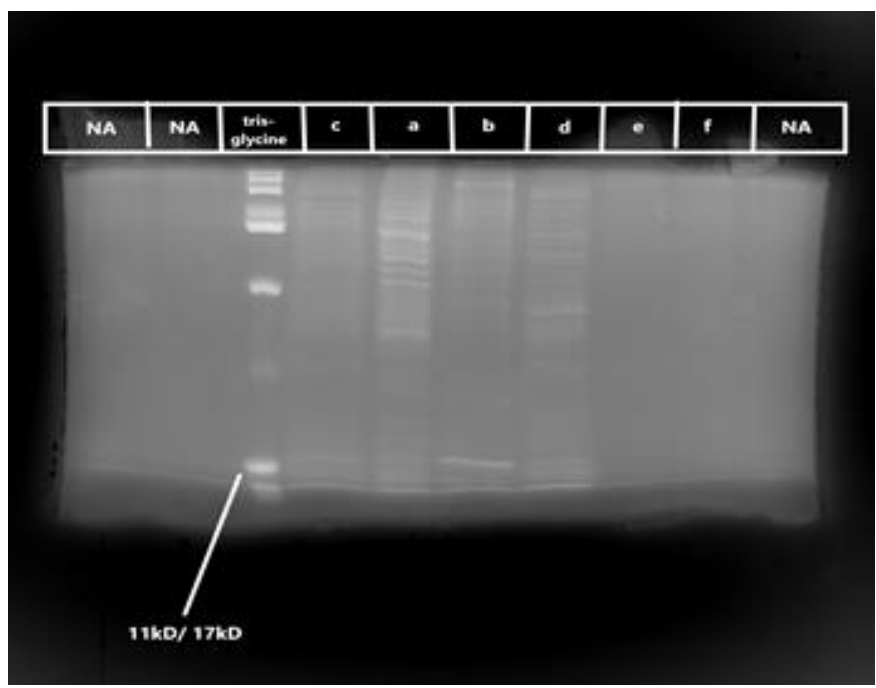

Figure 33

Table 2
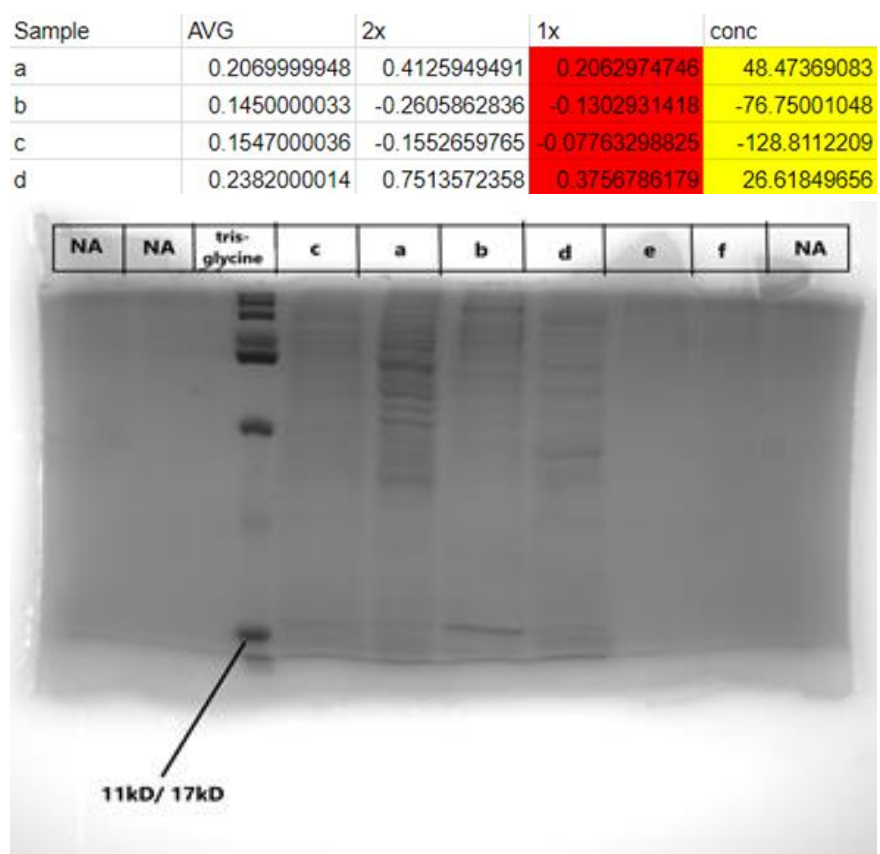

Figure 34

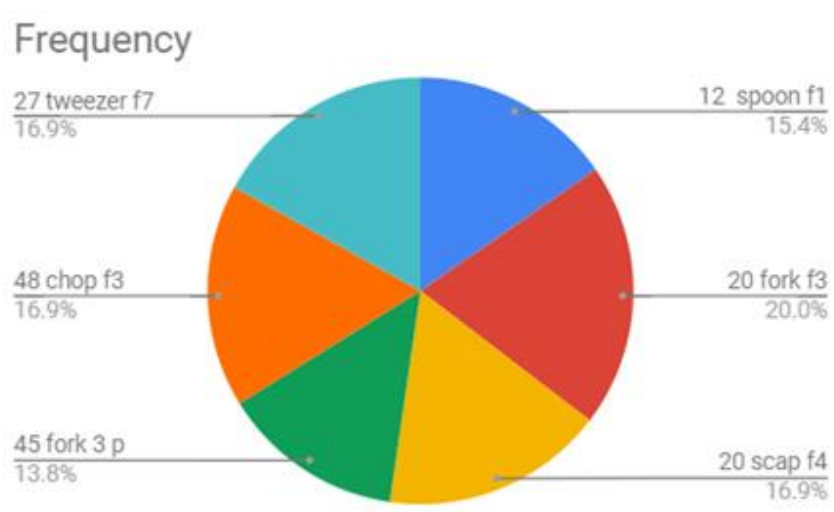

Figure 35 
Figure 36 and Figure 37 show a final analysis between frequency of all the generations over hundred seed values and microevolutionary forces observed in selected male and female bugs respectively.

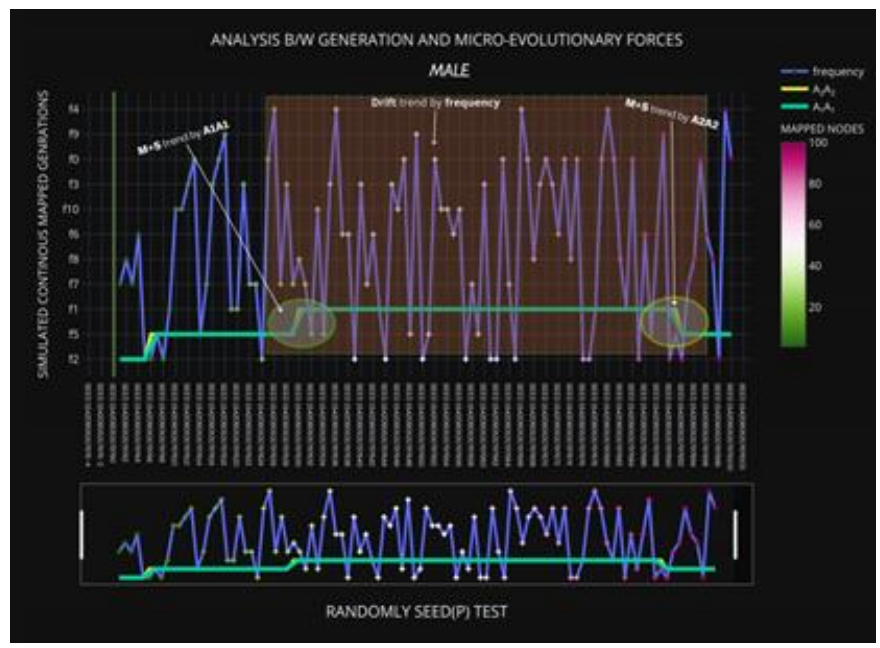

Figure 36

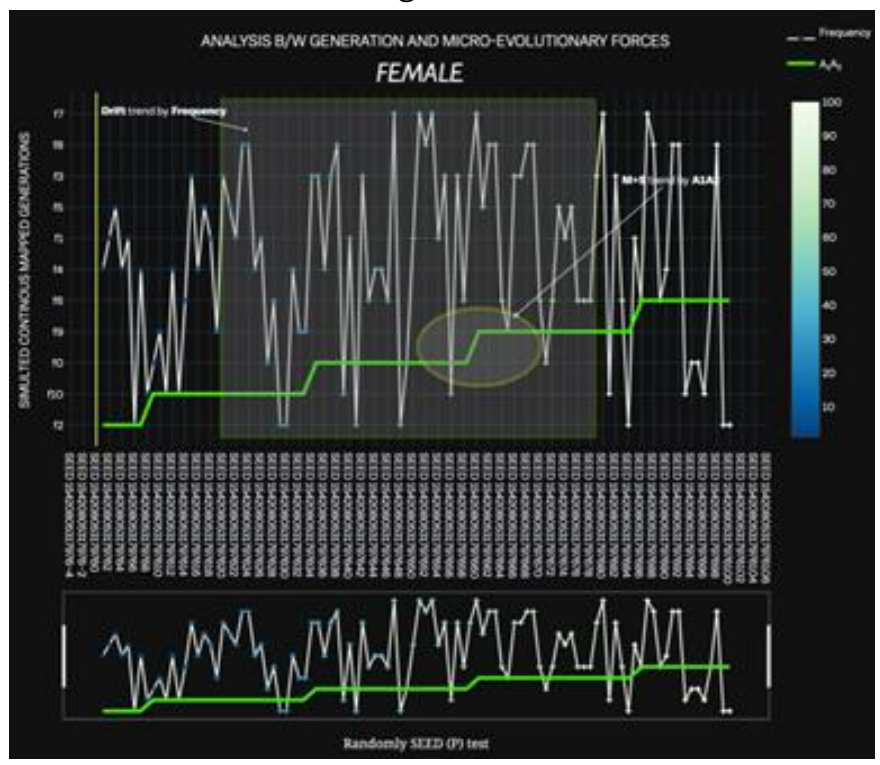

Figure 37

The above result was verified with the help of computer models generated for 2000 generation of pill bugs based on real frequency data. Considering the data for male bugs the following key is used for the graphs given belowFigures 38-44. The colour red and blue depict the main genotype frequency presenting selected length of survivors and victims respectively. The colour black represents A.1A1 and the colour purple represents A1A2. The color light brown represents A2,A2. Figure 38 shows 1000 generations for male bugs based on our readings. Figure 39 depicts the establishment of genetic drift and speciation after 1000 generations based on our reading. This acts as an evidence for the result obtained by our analysis. Figure 40 establishment of genetic drift with single dominant genotype. Figure 41 represents establishment of genetic drift with one dominant and one recessive genotype. Figure 42 shows the complete removal of homozygous recessive genotype from the population. Figure 43 shows a comparison between genotypes with one dominant and both dominant traits. Figure 44 shows that after 1000 generations there was a complete removal of victims along with the formation of a new species. This clearly indicates that it is a slow evolutionary process showing bottleneck effect therefore the microevolutionary forces mutation and selection become evident.

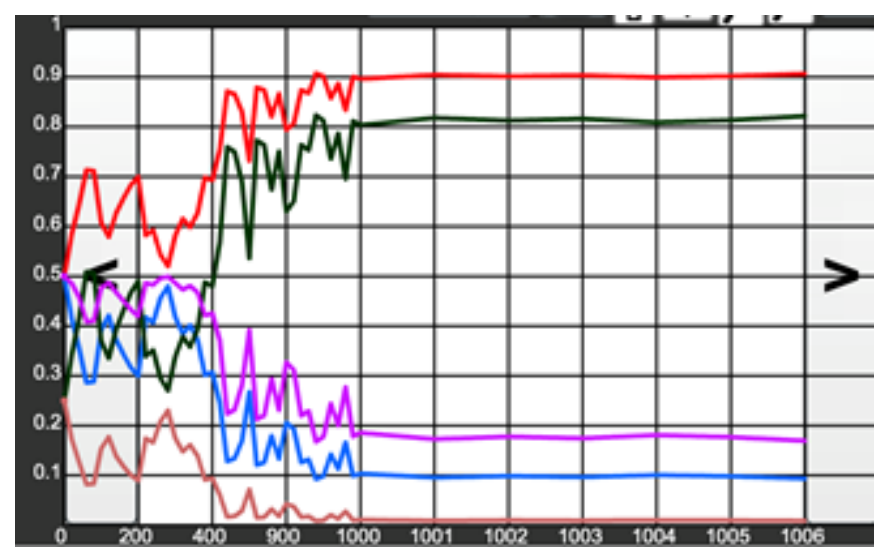

Figure 38

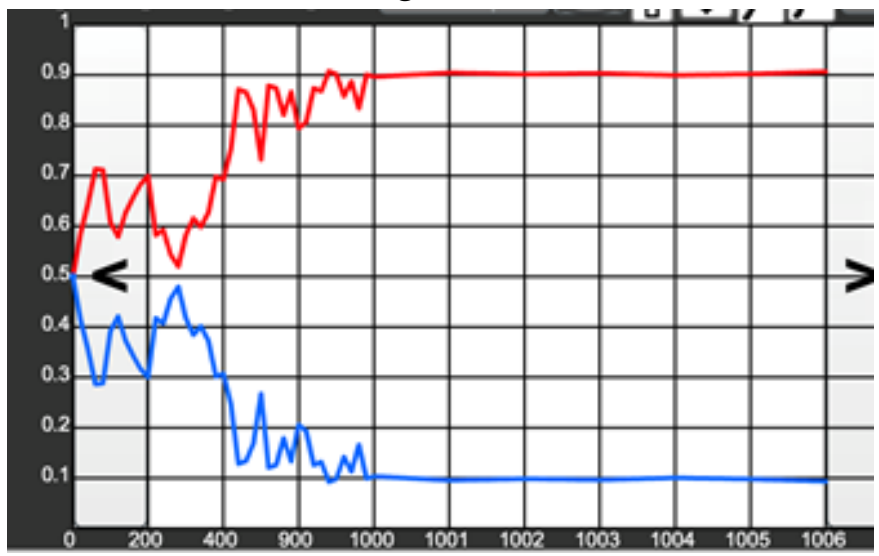

Figure 39 


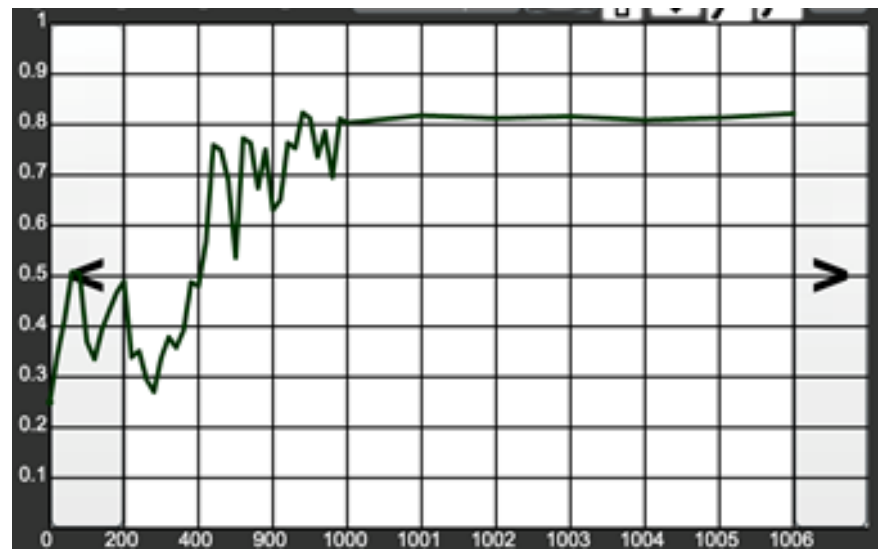

Figure 40

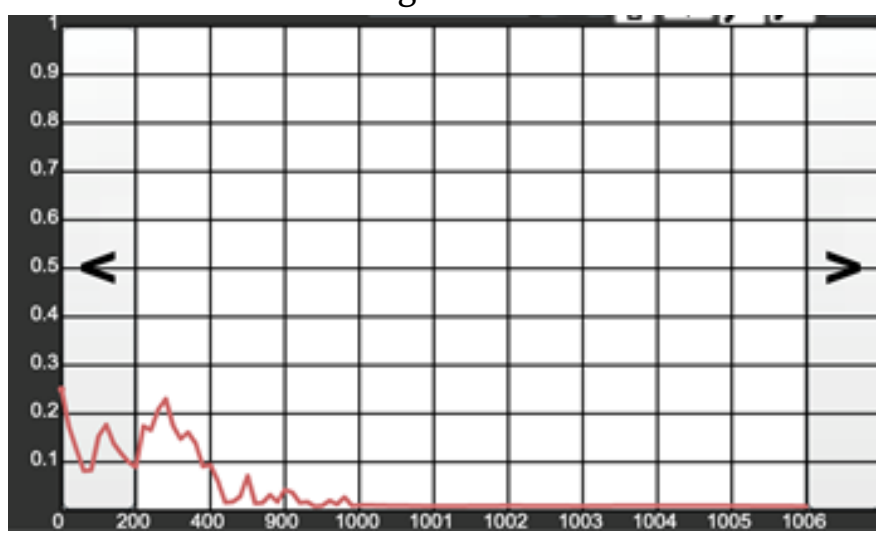

Figure 41

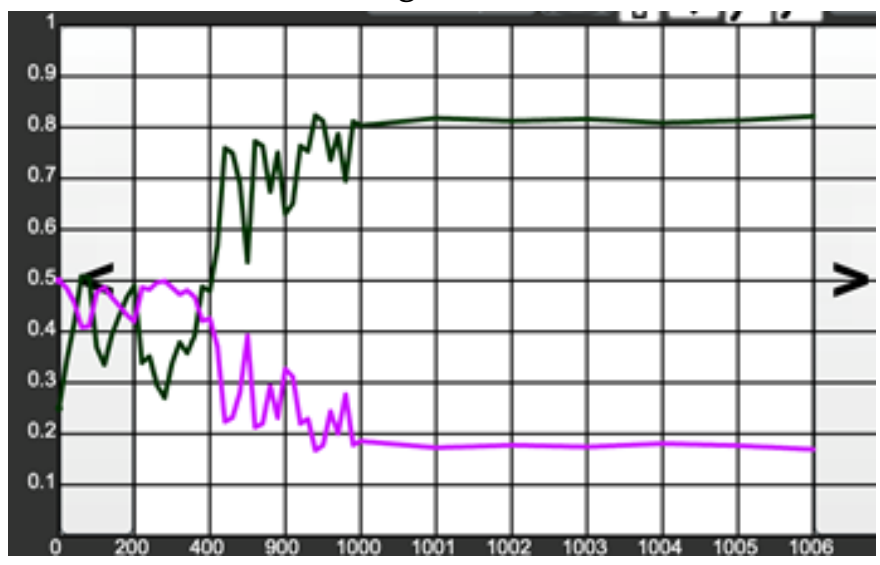

Figure 42

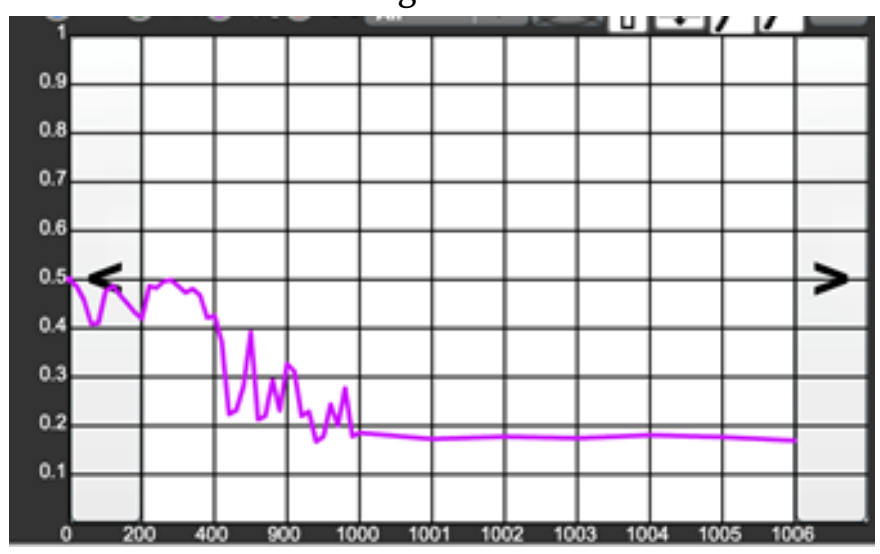

Figure 43

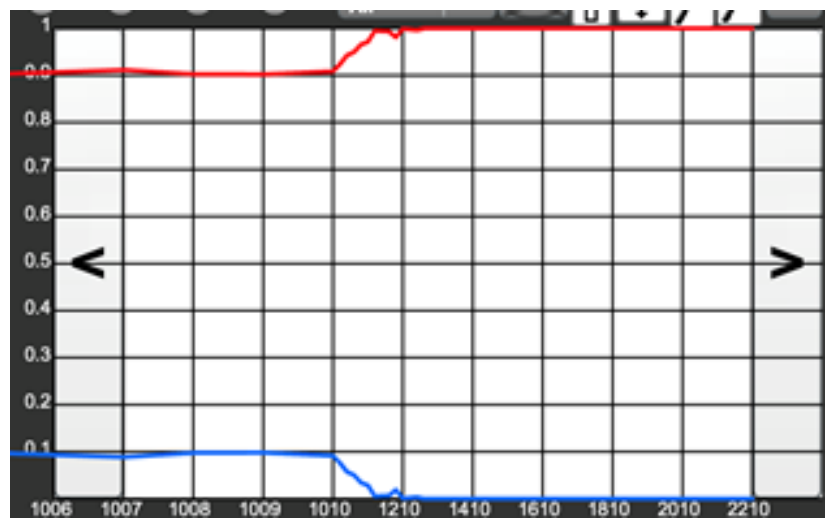

Figure 44

Similarly Figures 45-50 represent the computer model for female bugs with the difference that the character considered was weight unlike length that was considered in males. Consider Figure 51 it becomes evident that unlike males the formation of new species and complete removal of victims took place after 2000 generations.

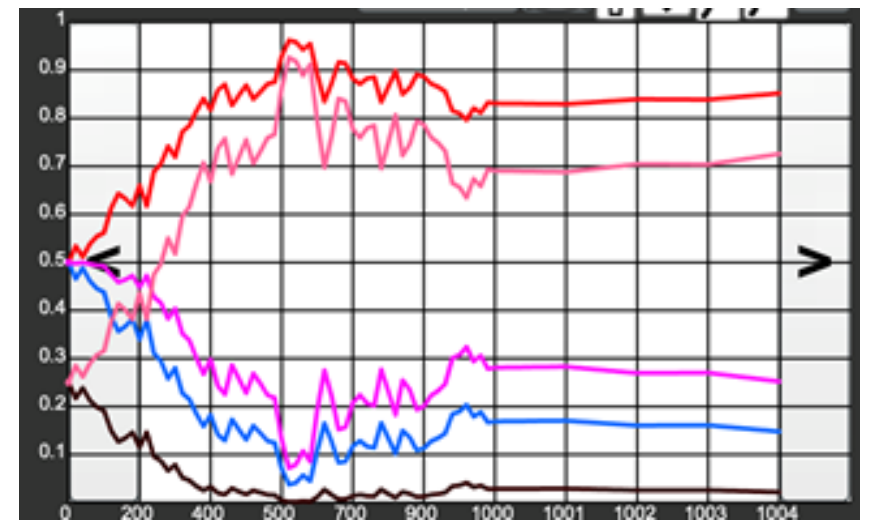

Figure 45

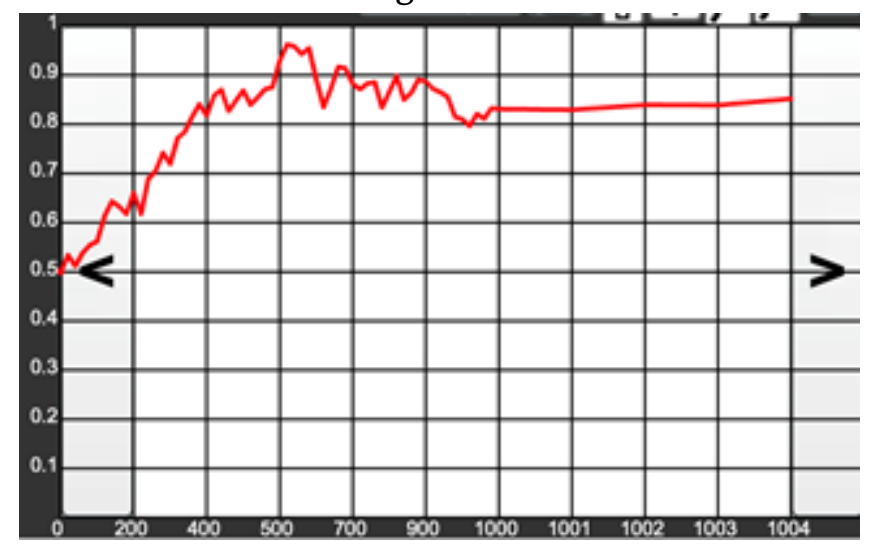

Figure 46 


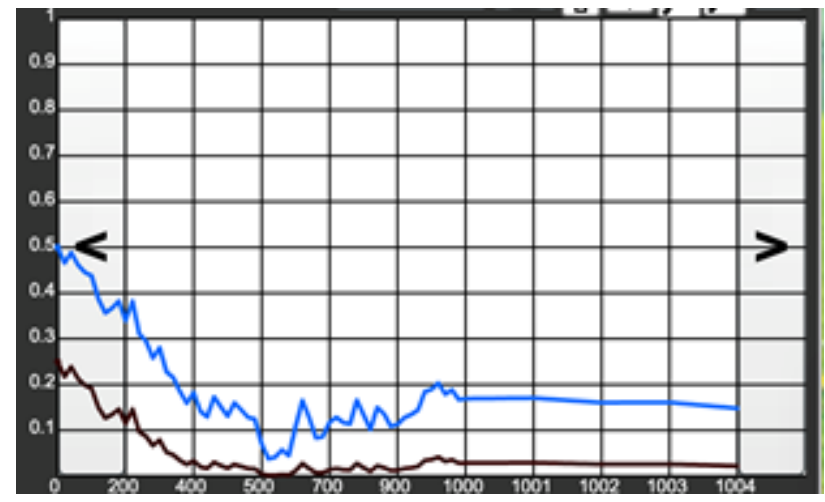

Figure 47

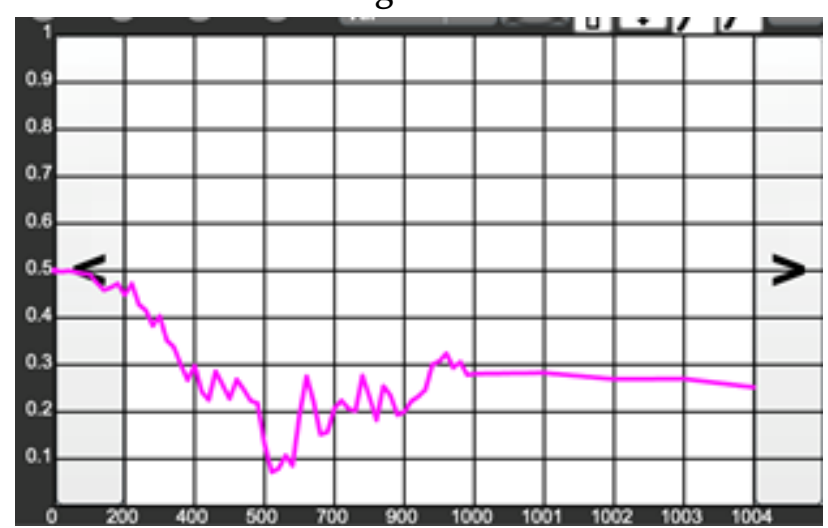

Figure 48

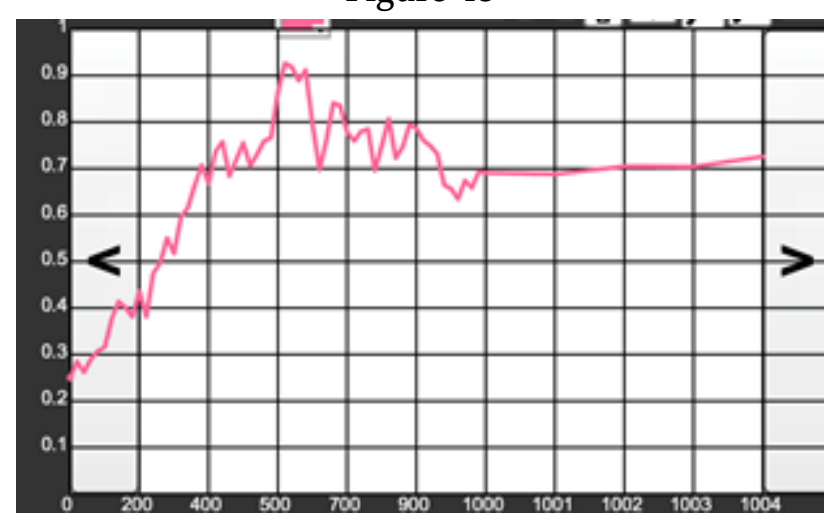

Figure 49

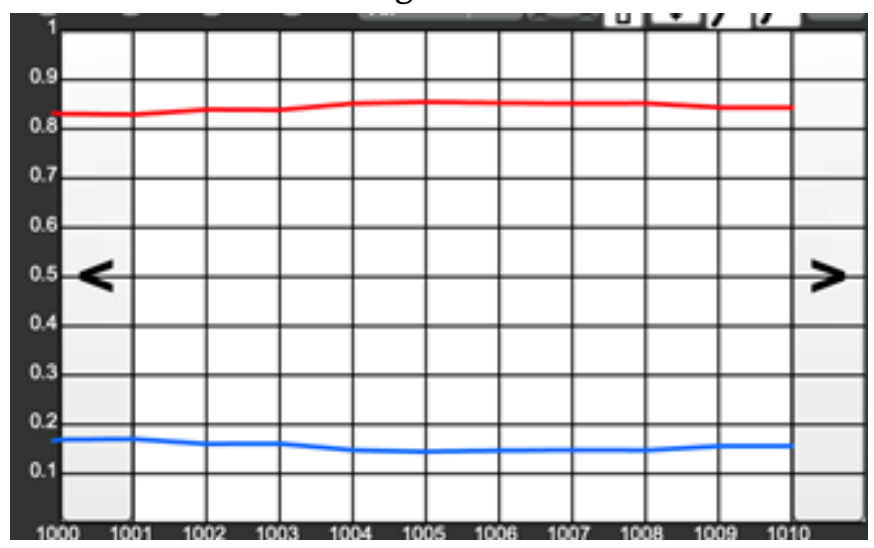

Figure 50

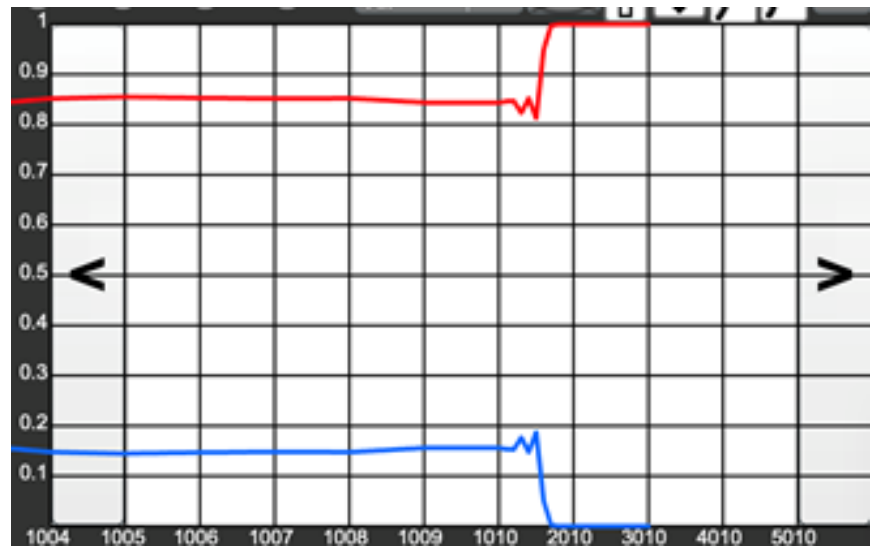

Figure 51

These predicted computer models verify our analysis and we can conclude that the microevolutionary forces operating over generation for the bugs were genetic drift and mutation and selection.

The bugs of the parent generation belonged to two different origins Portugal and UkraineFigure 52.The bugs were further analyzed to study adaptive radiation by selecting the fittest bug from each generation.
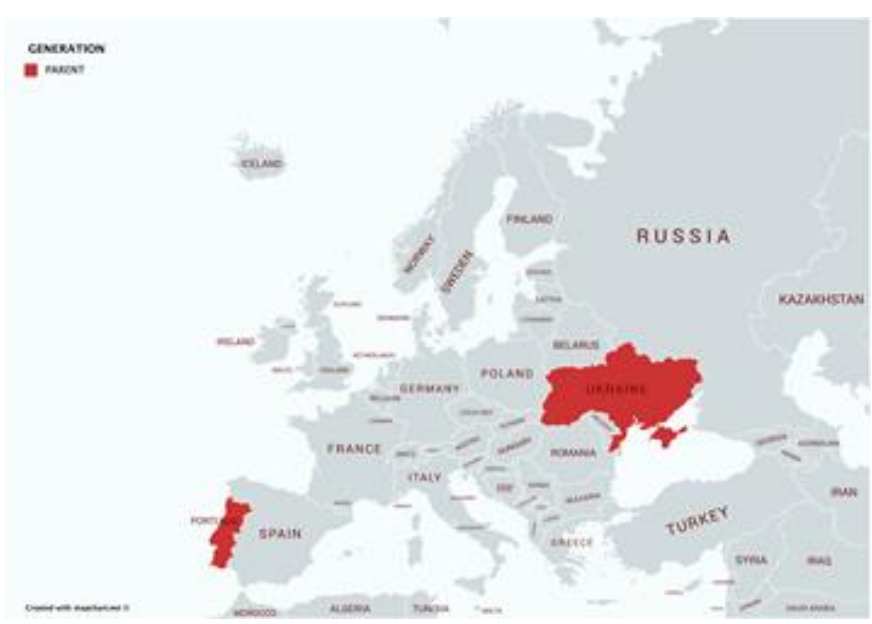

Figure 52

Once the selection was made, their DNA was isolated using the above defined method and was digested with restriction enzymes namely but not limited to EcorV, EcorI, BamHI, EacI, XhoI, Hind III etc. Further, electrophoresis was performed and each 
pattern obtained was compared to the gene sequence of parents from both the origins Ukraine and Portugal. Figures 53-55 represent the above result. The comparison was standardized with $1 \mathrm{~kb}$ ladder.

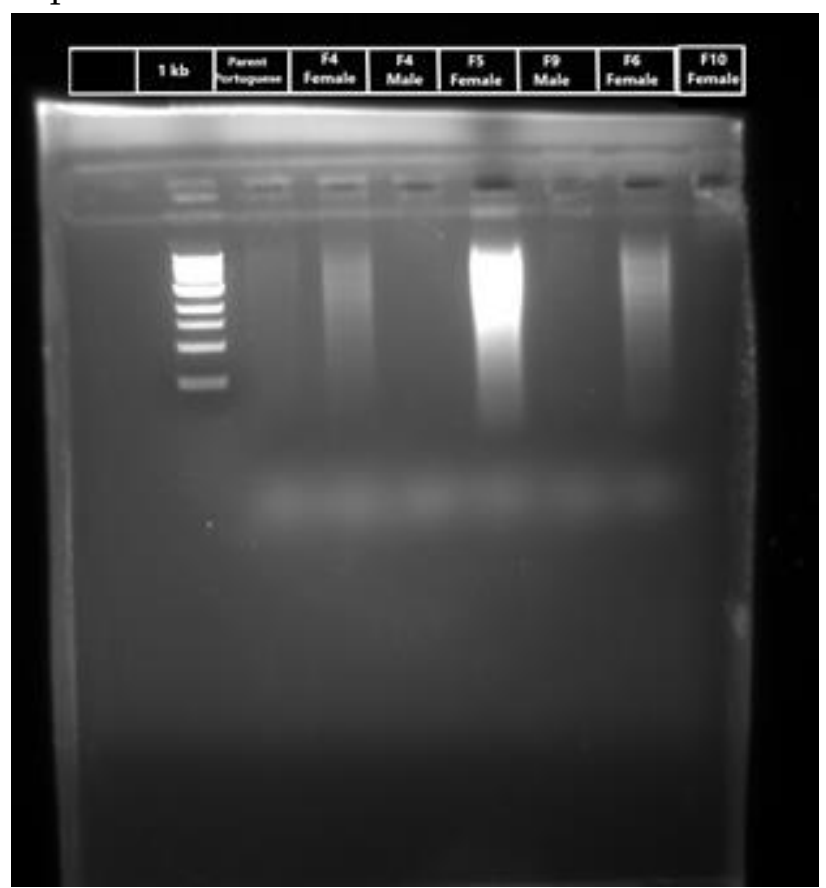

Figure 53

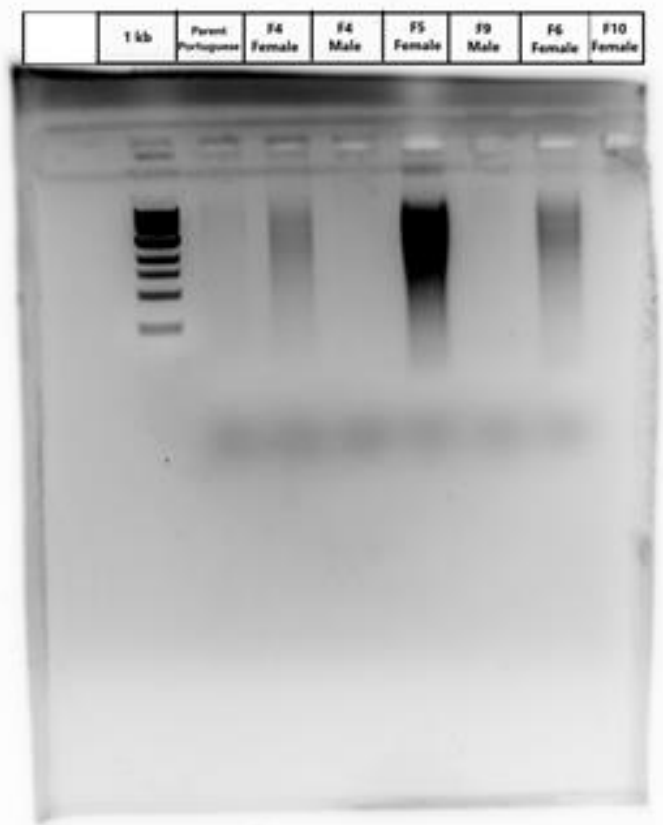

Figure 53.1

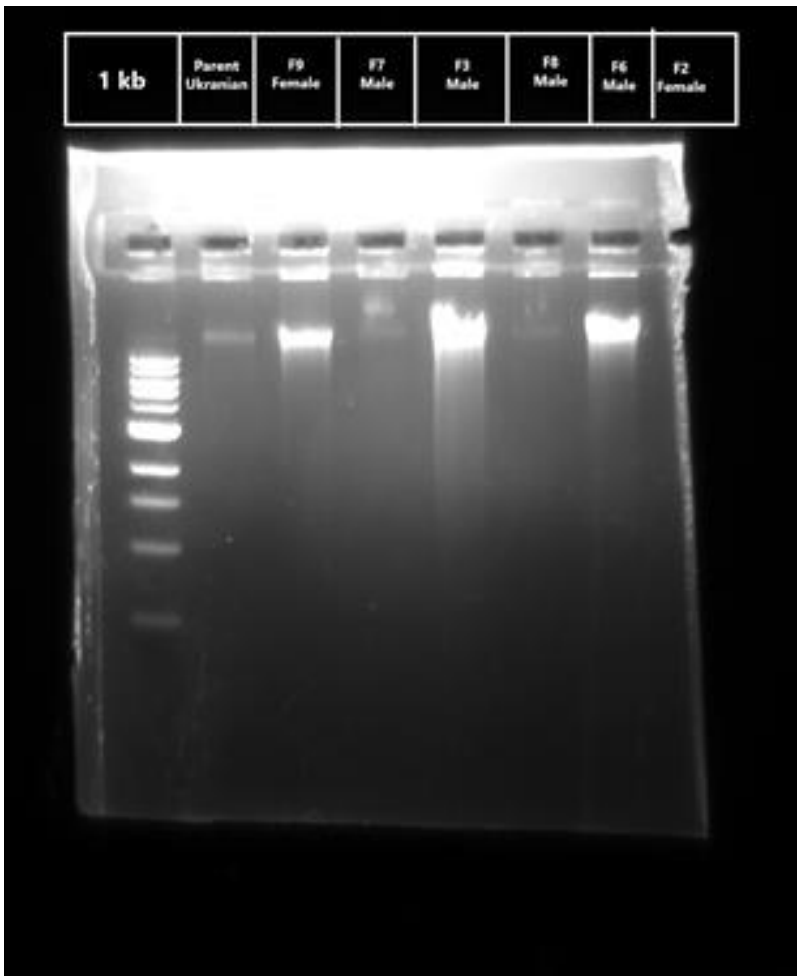

Figure 54
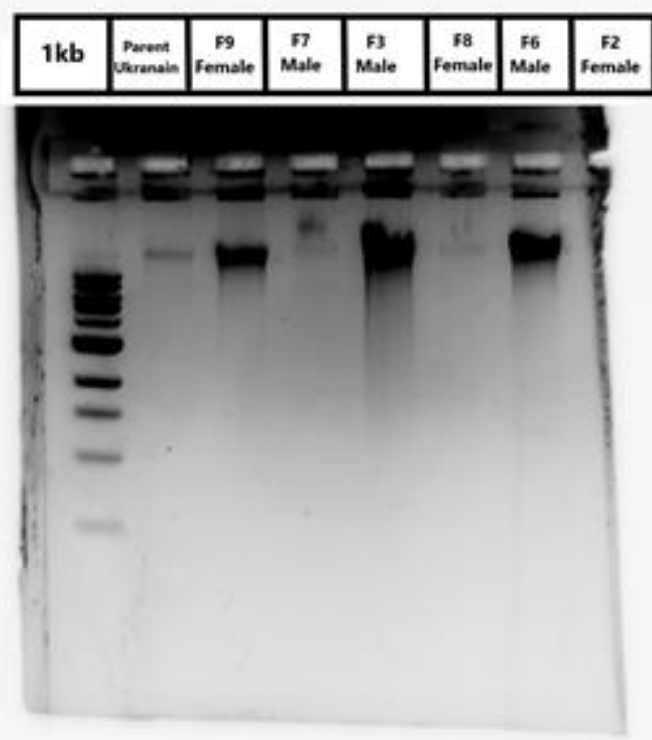

Figure 54.1 


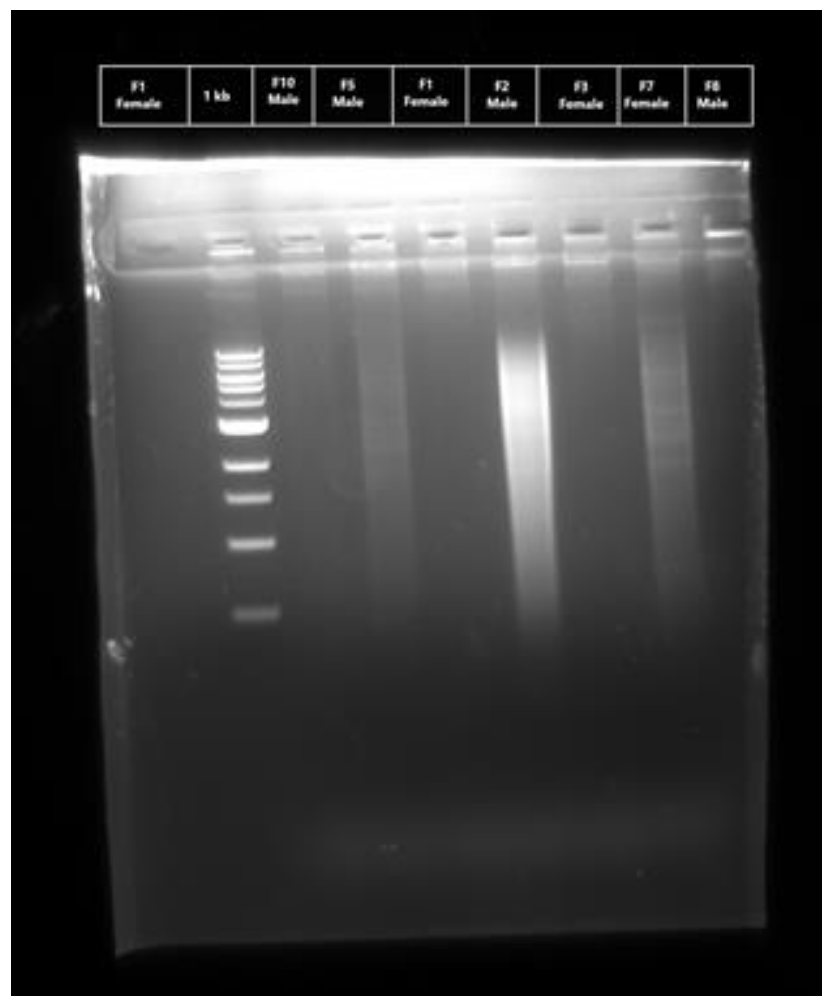

Figure 55

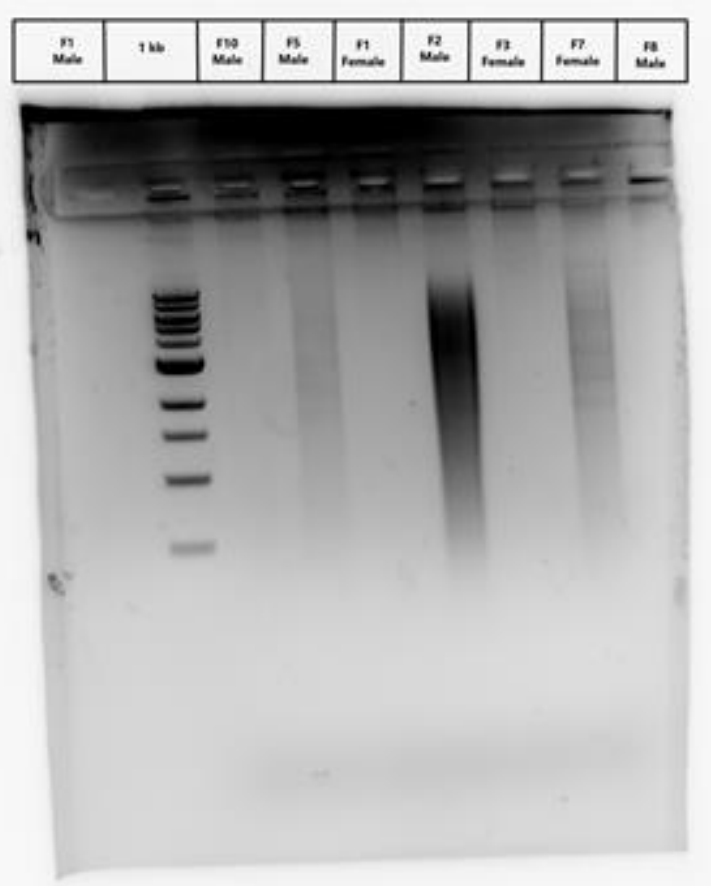

Figure 55.1

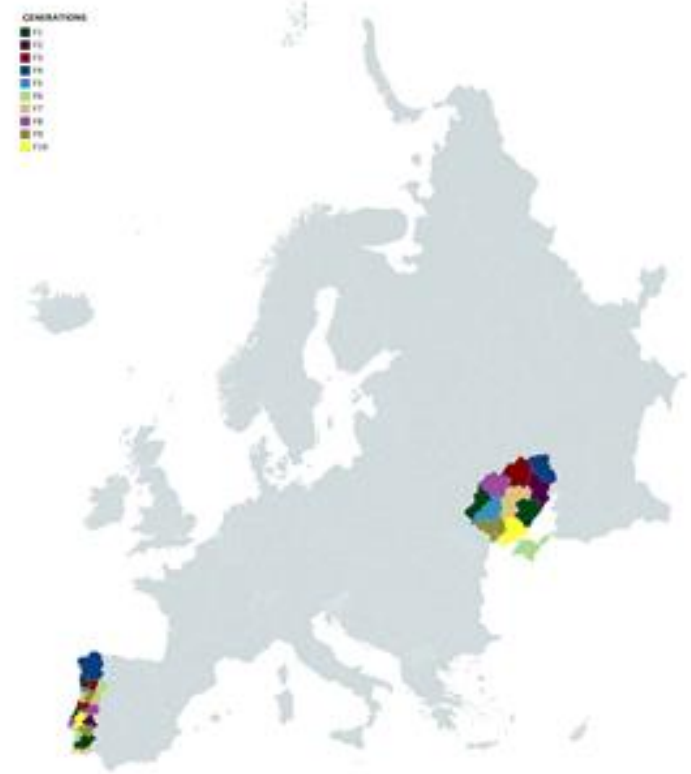

Figure 56

The same result was obtained by computer models.Figures and show the computer model for The parent generation belonging to the origin Ukraine and Portugal respectively. Figure show the computer model for male bugs whereas Figures show the computer model for female bugs. Figure shows the final prediction of radiation shown by the 10 generations, clearly showing the transfer of genes.

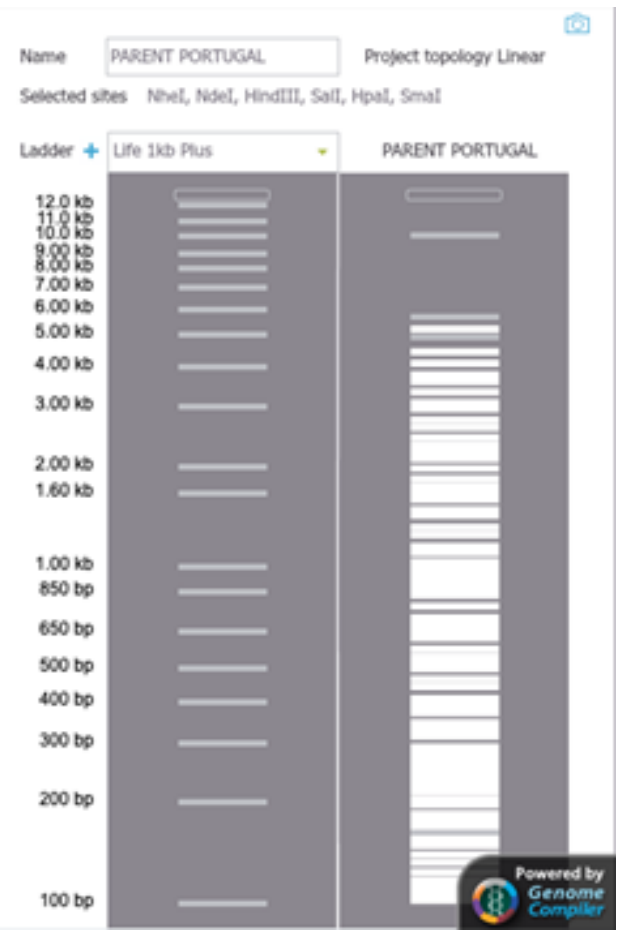




\section{Figure 57}

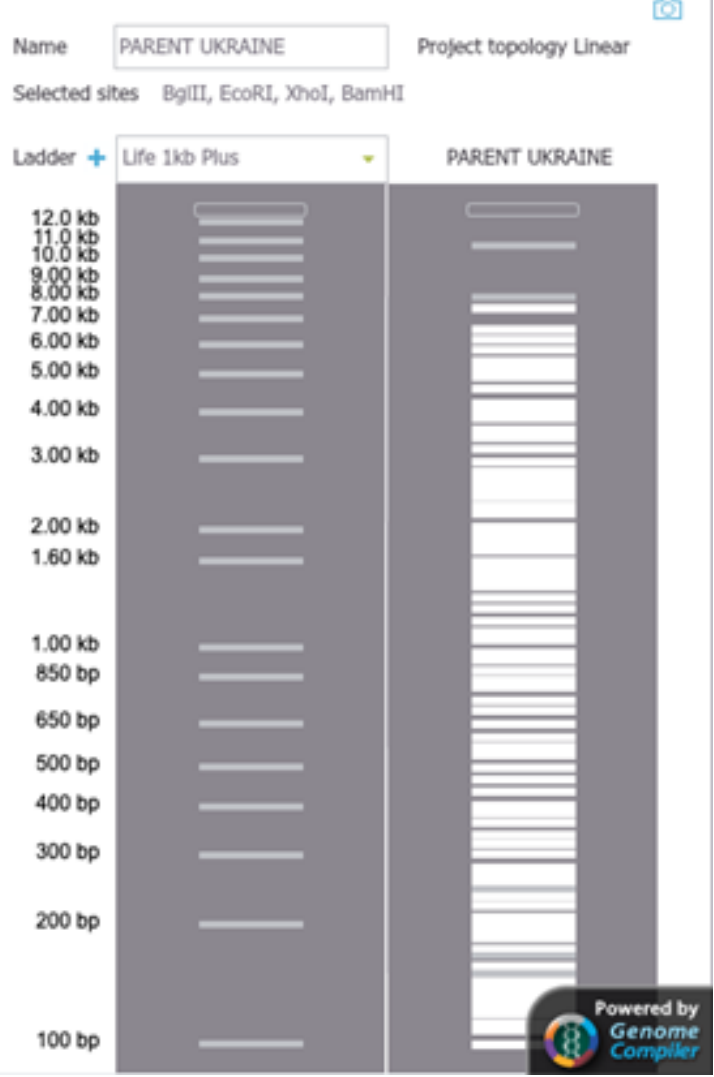

Figure 58

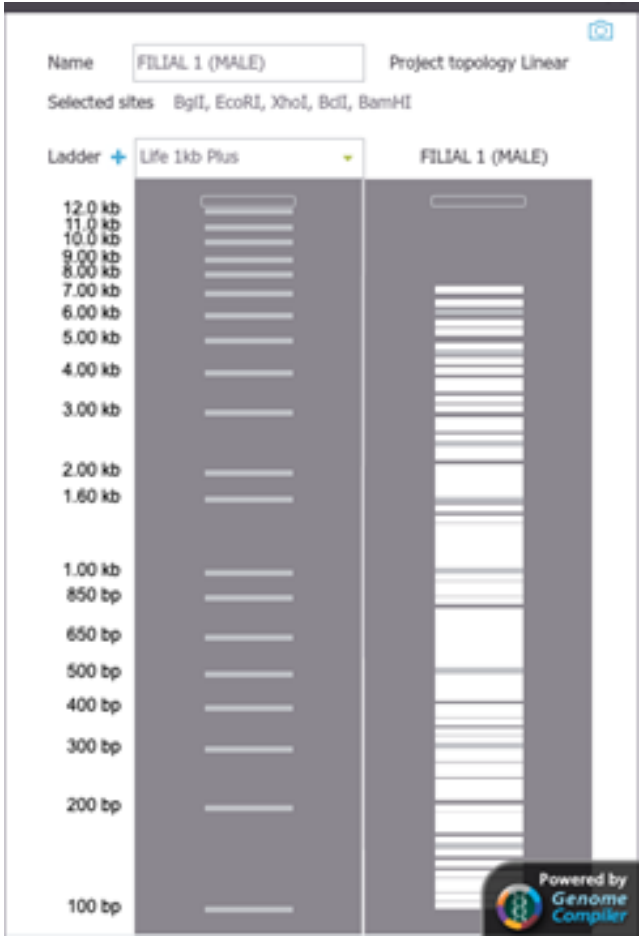

Figure 59

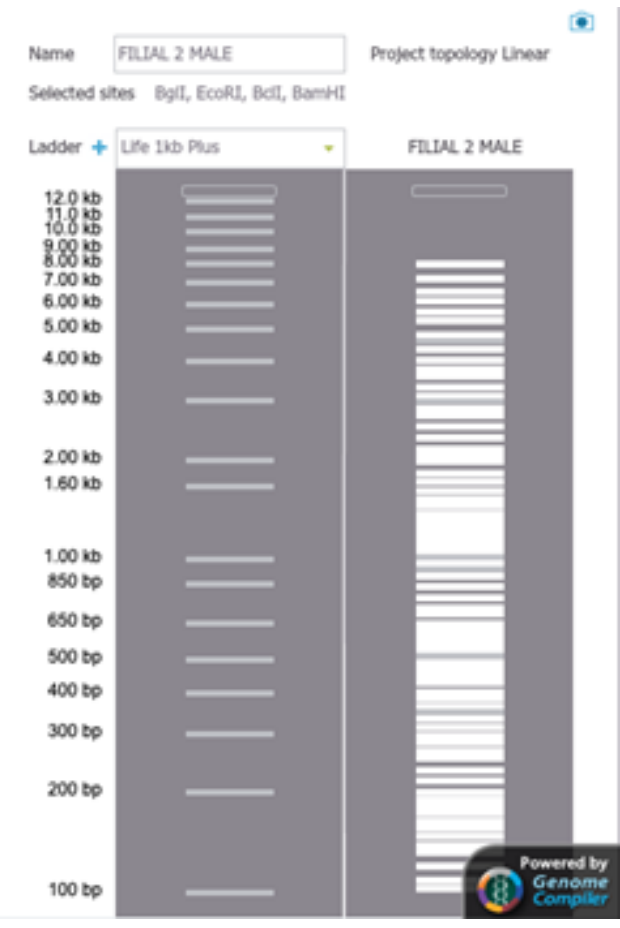

Figure 60

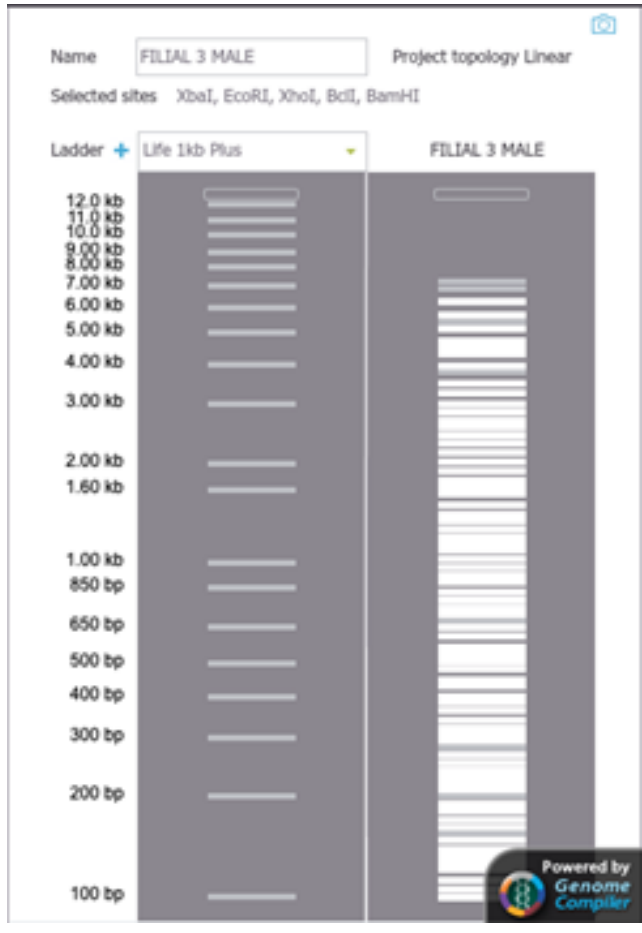

Figure 61 


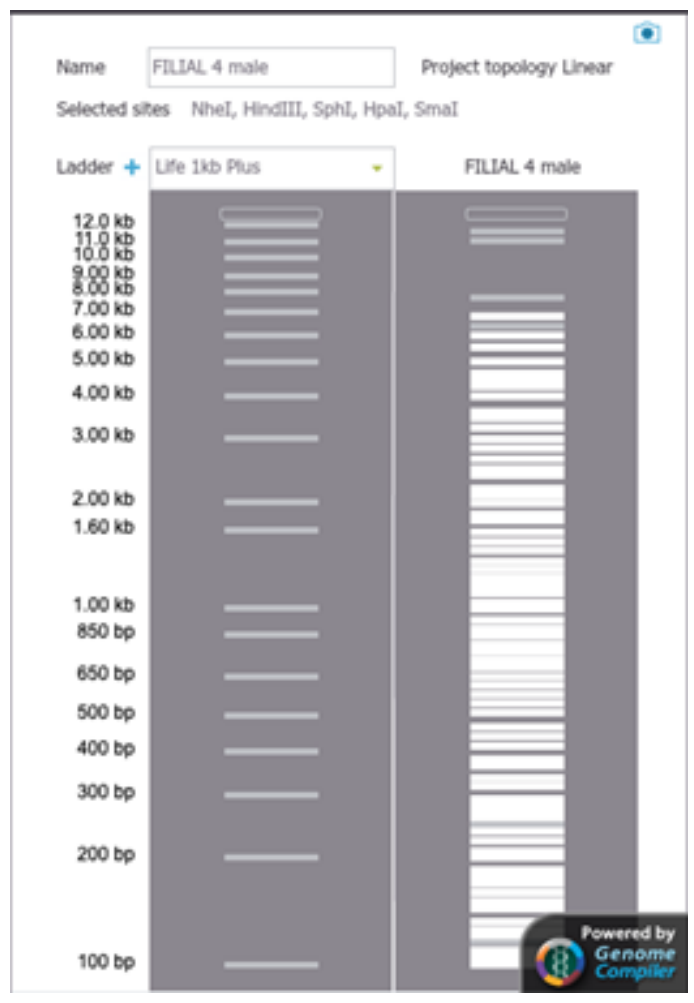

Figure 62

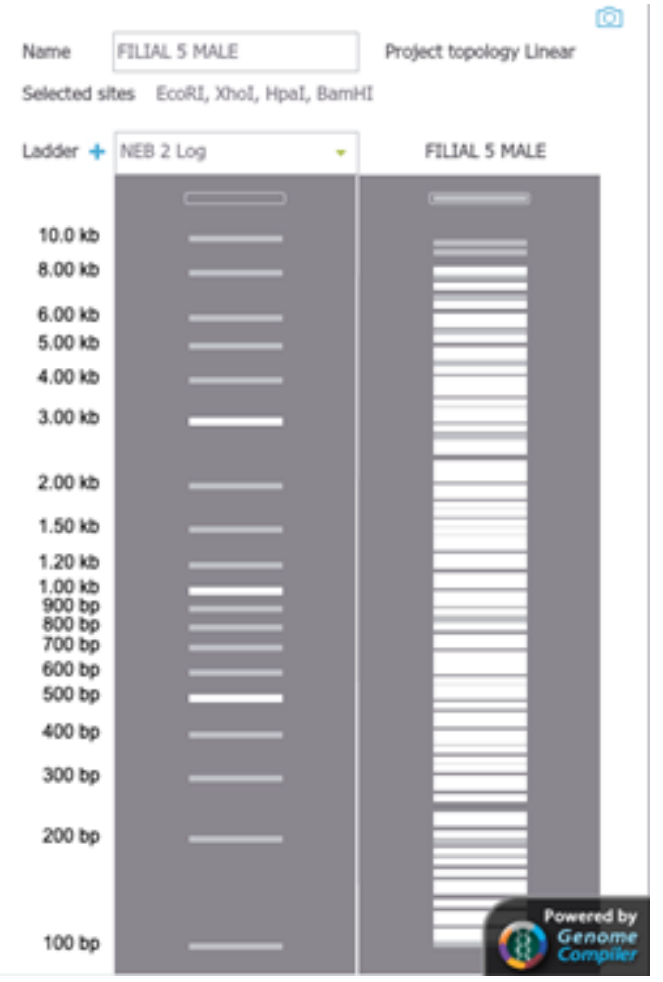

Figure 63

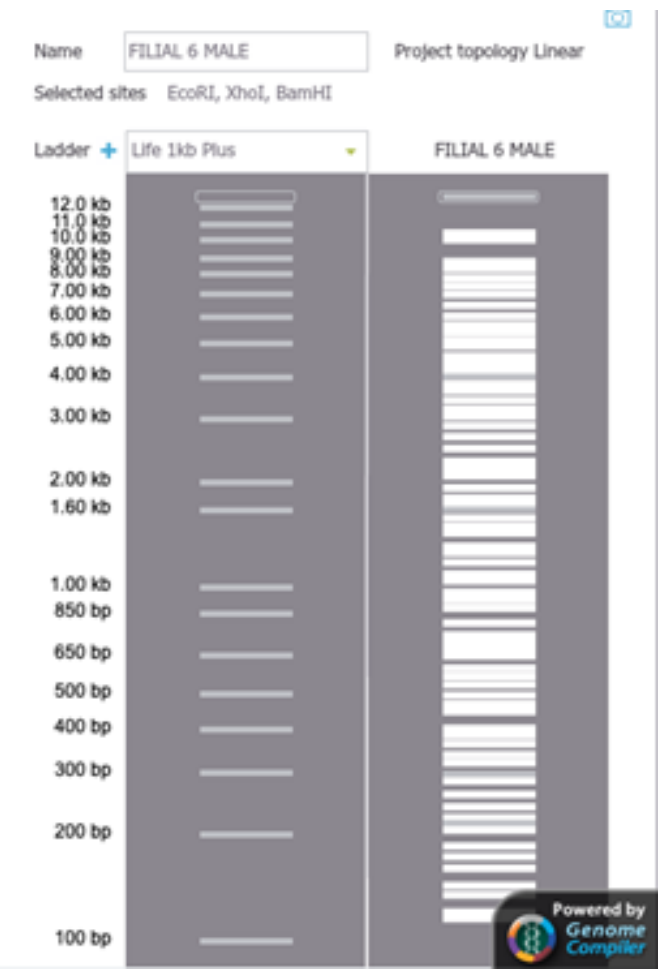

Figure 64

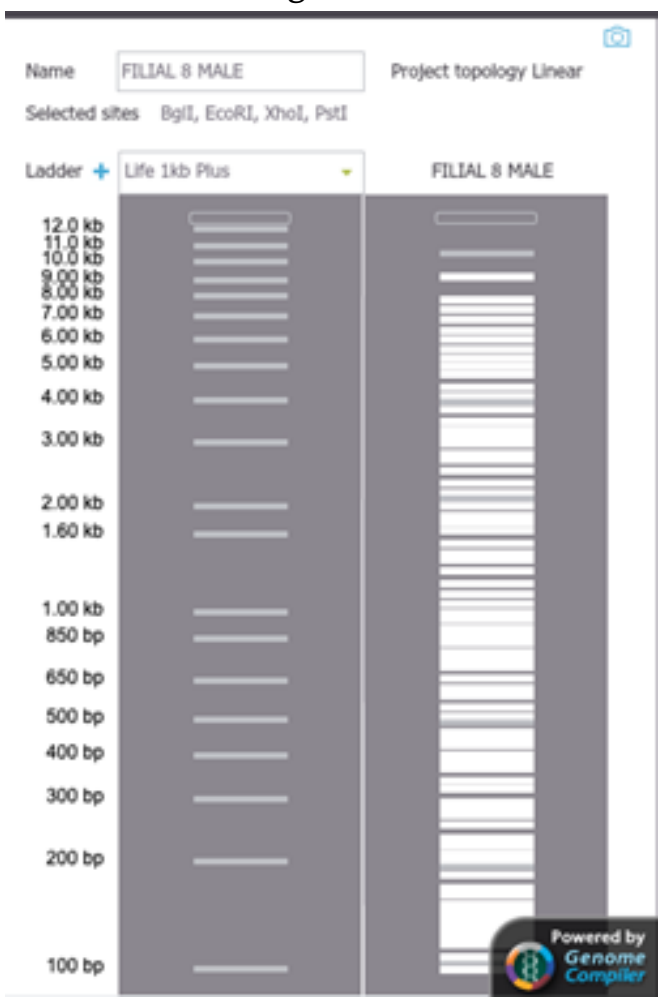

Figure 65 


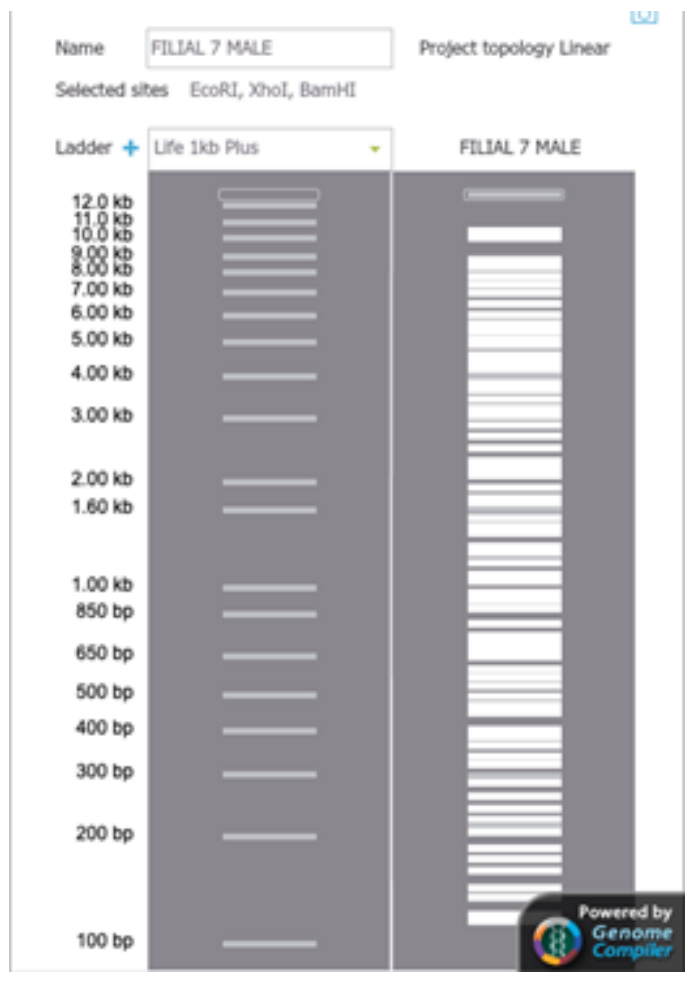

Figure 66

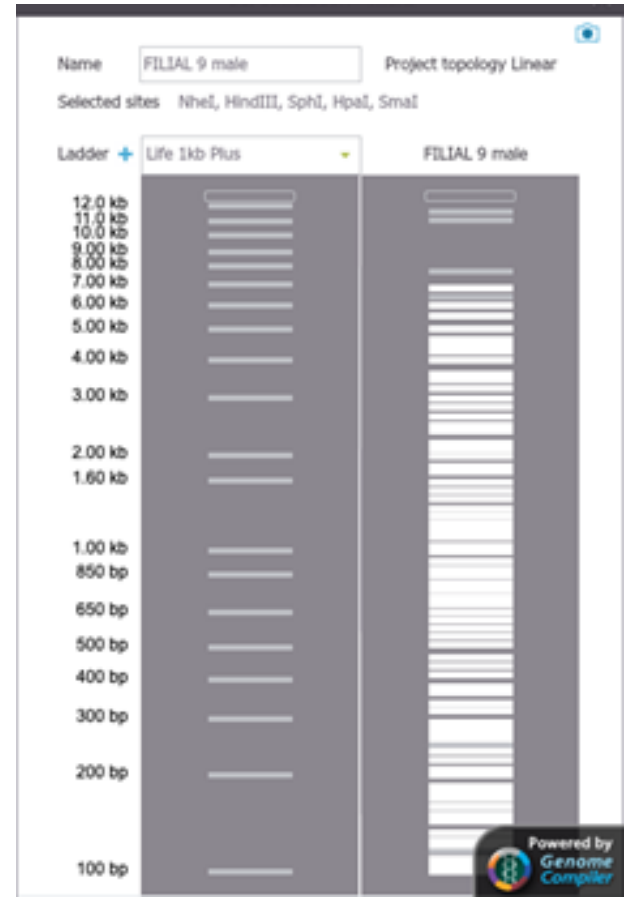

Figure 67

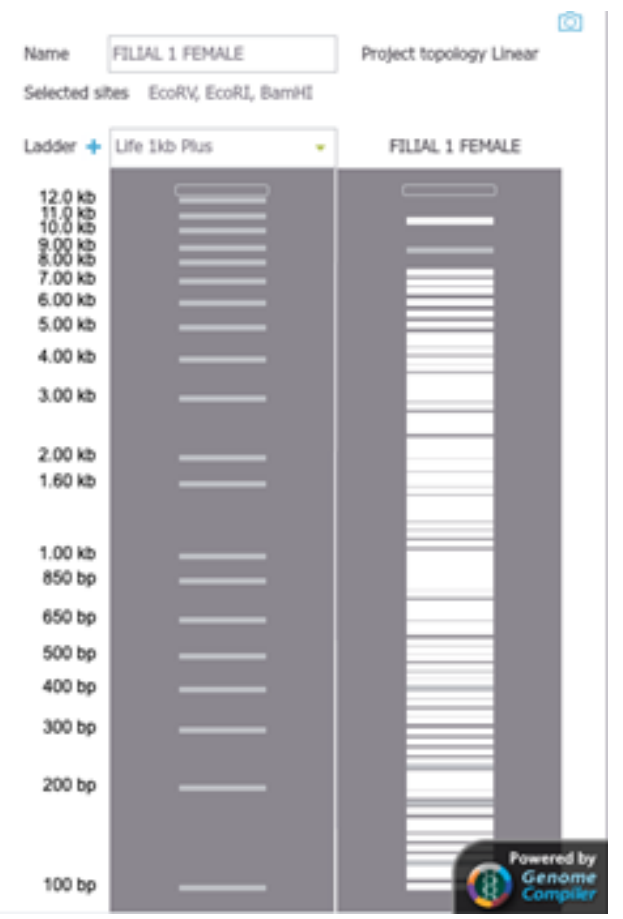

Figure 68

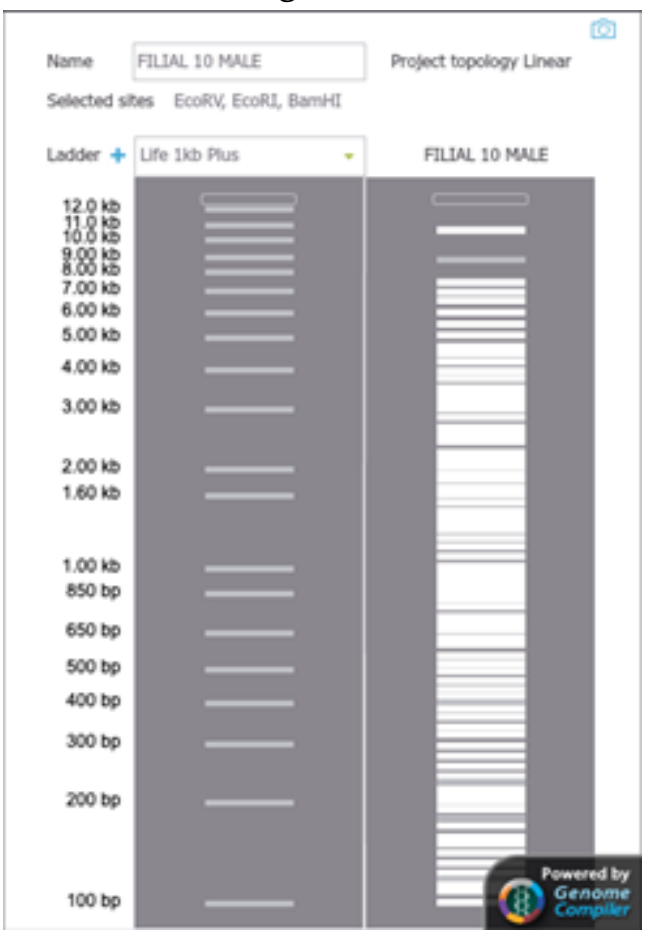

Figure 69 


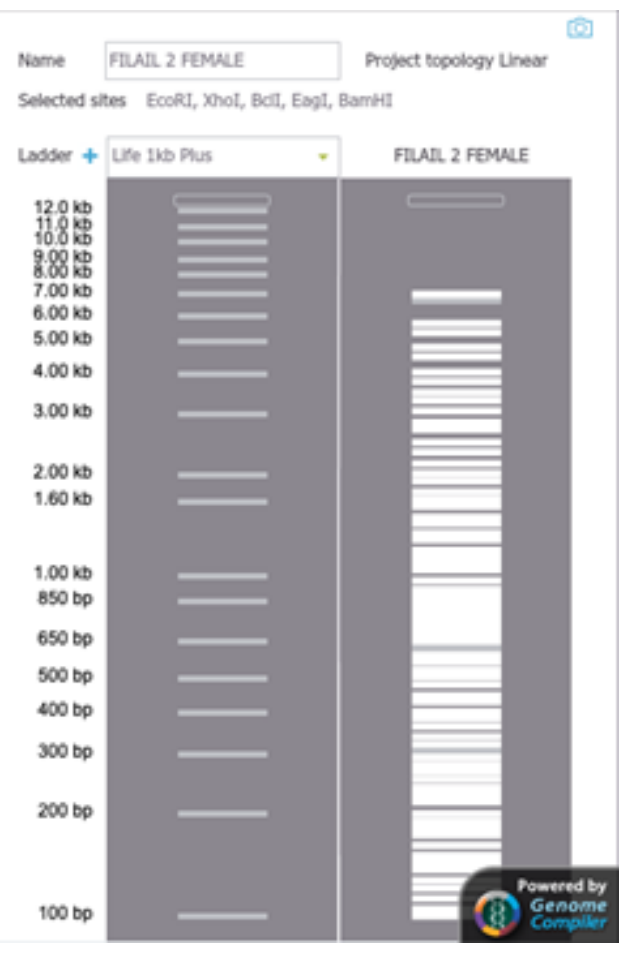

Figure 70

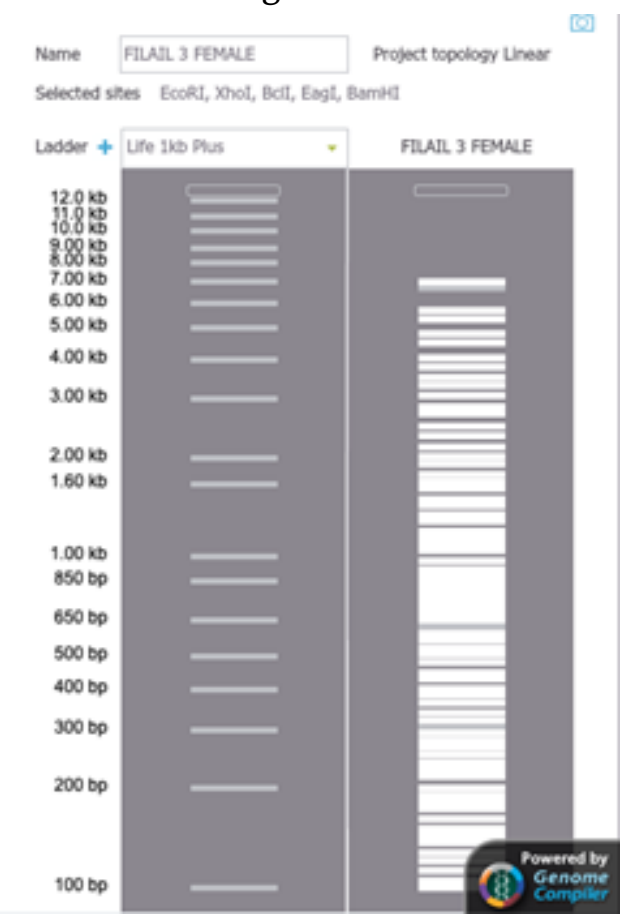

Figure 71

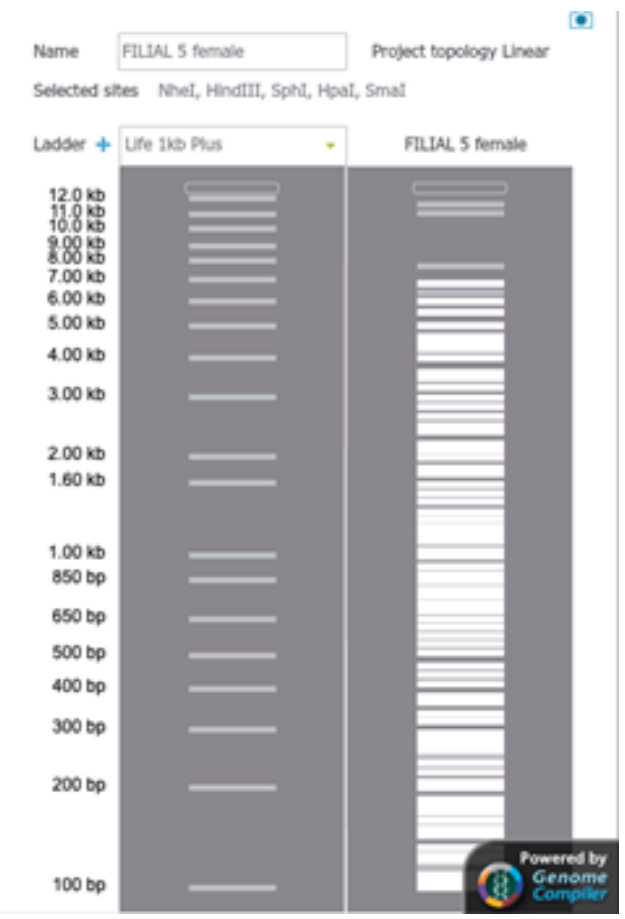

Figure 72

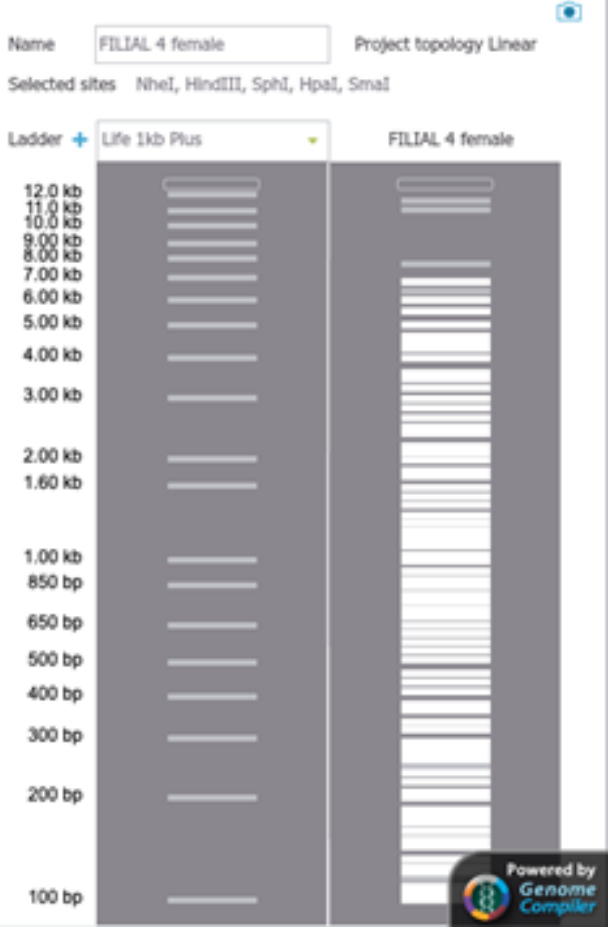

Figure 73 


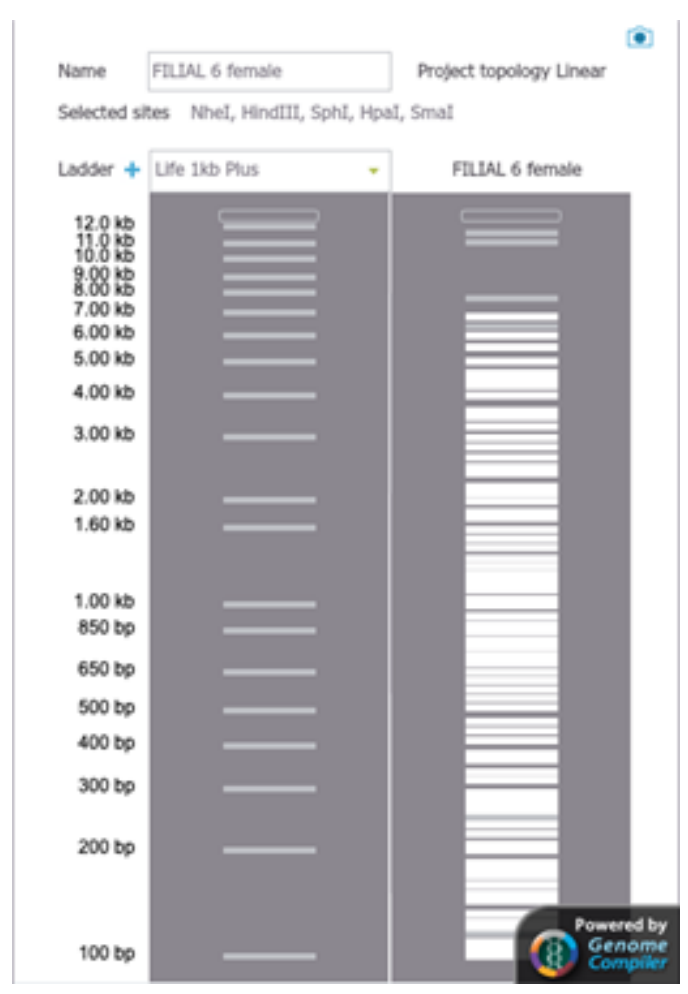

Figure 74

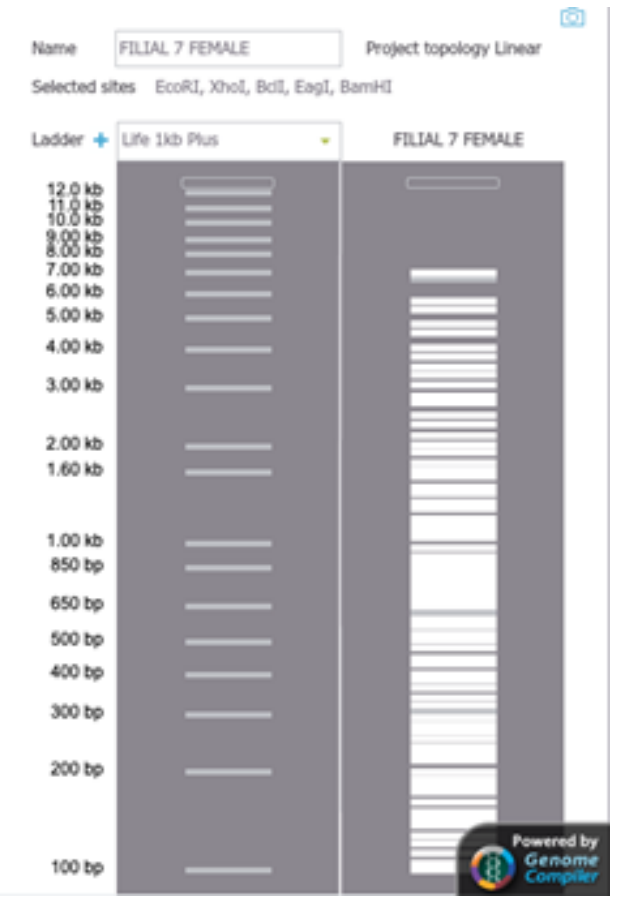

Figure 75

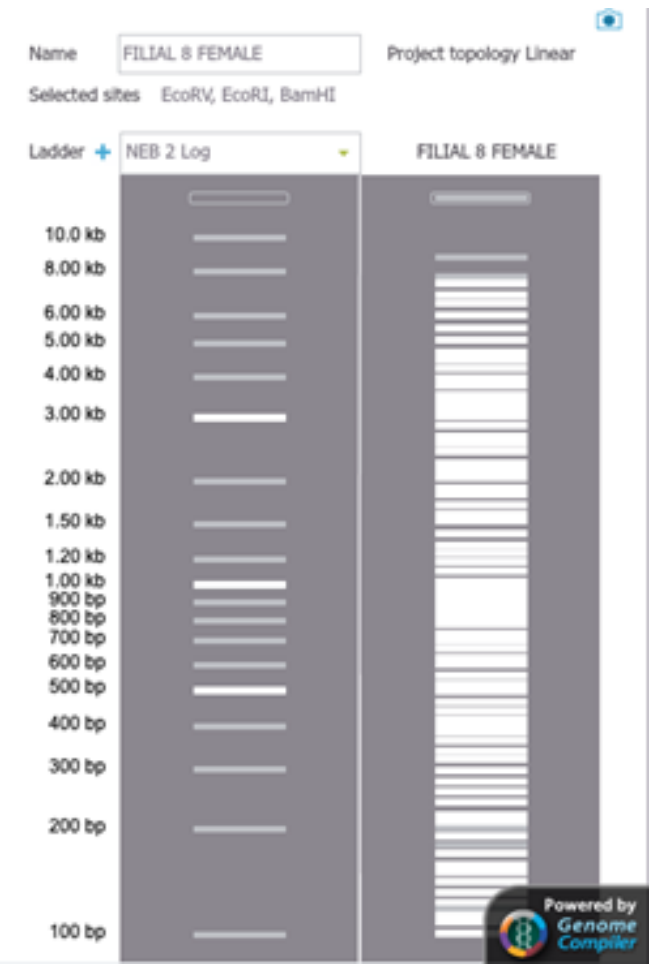

Figure 76

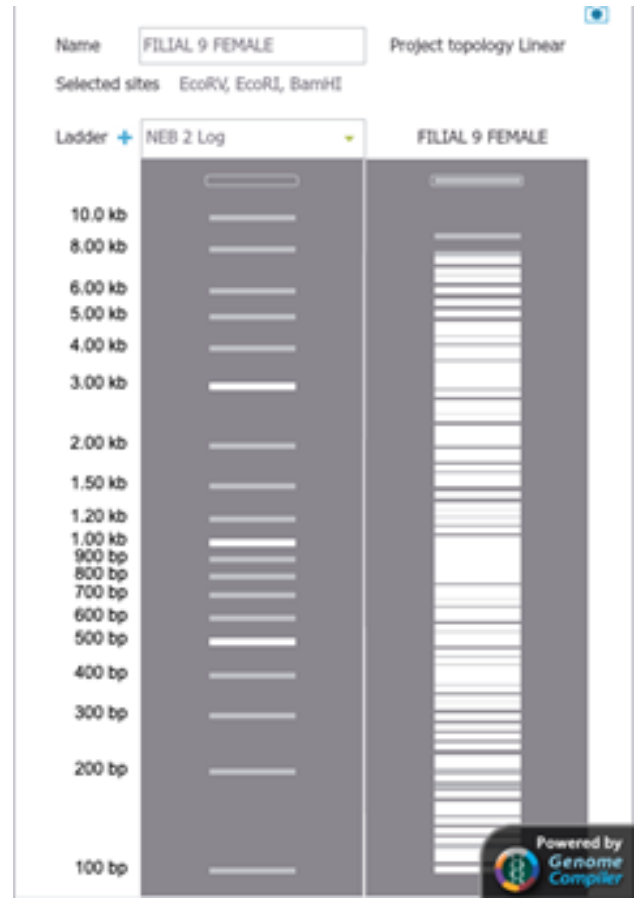

Figure 77 


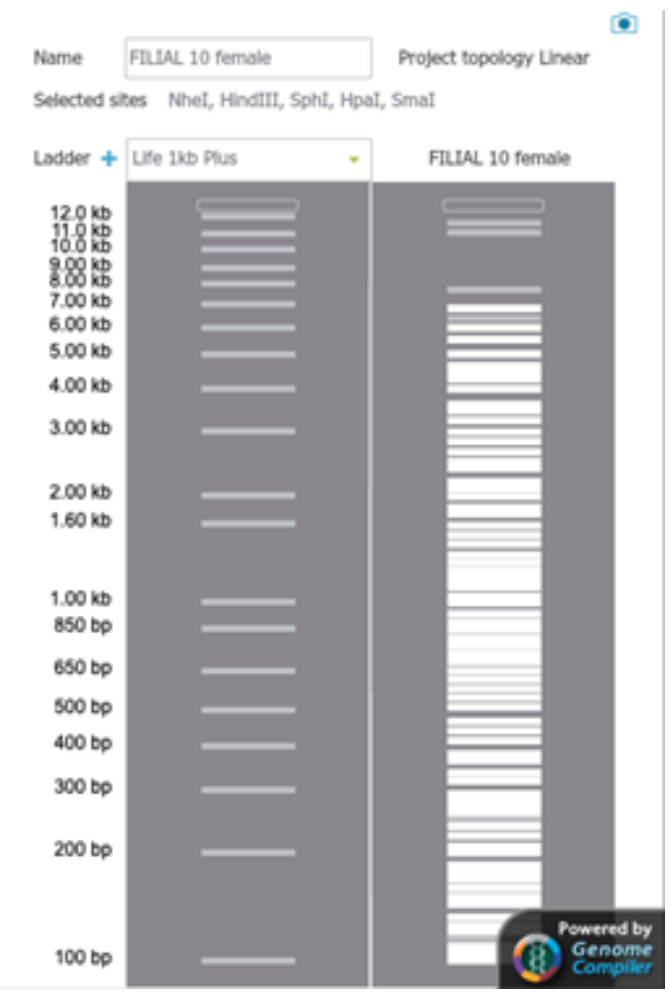

Figure 78

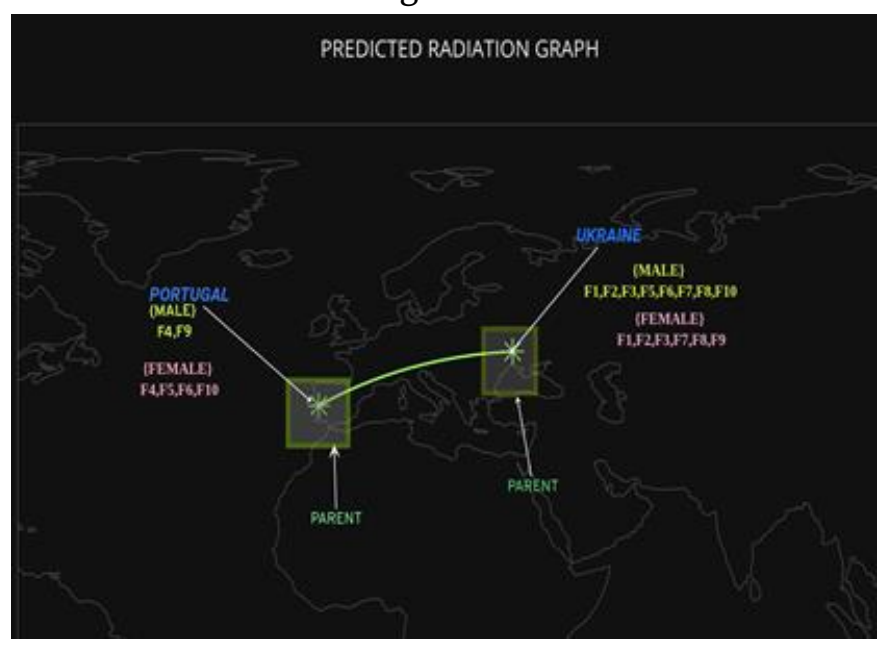

Figure 79

Considering all the observed results, a formula for linking natural selection with genetic drift and further adaptive radiation was derived using the previously described method.

Analysis using Punnett square

Results obtained from observed values from all generations

Ukraine: Portugal = 23:21

Results derived from general Punnett Square
Ukraine: Portugal = 13.5: 4.5

Equating both the results

$13.5 / 18=23 \mathrm{a} / 44 ; 4.5 / 18=21 \mathrm{~b} / 44$

$\mathrm{a}=1.4347 \mathrm{~b}=0.523$

$\mathrm{a} / \mathrm{b}=2.7432(\mathrm{a} / \mathrm{b}=$ multiplying factor used in the final equation while equating with regression models)

Analyzing the relation between length and weight of bugs by initially crossing WWLL ${ }^{*}$ wwll and later $\mathrm{WwLl}^{*} \mathrm{WwLl}$; considering more weight (W) and less height as dominant (L). The bugs belonging to origin Ukraine were WWLL and that to Portugal were wwll. The first cross was made between WWLL* wwll all the observed outcomes were WwLl. Then crossing $\mathrm{WwLl} *$ WwLl the results were analyzed to obtain the percentage of bugs showing the phenotype of heavyweight and less height which was the observed phenotype of selected bugs which were selected using simulated natural selection.

Required phenotype percentage- $56.25 \%$

$23 / 44^{*}(56.25 / 100)=\mathrm{z} 1=0.2940 ; \mathrm{p} 2=29.40$

$21 / 44^{*}(56.25 / 100)=\mathrm{z} 2=0.2684 ; \mathrm{q} 2=26.84$

Using Hardy Weinberg Principle

$(\mathrm{p}+\mathrm{q}) 2=112.08$; where 1.1208 is the deviation

$2 p q=55.844$

From Regression Model

The following regression equations are obtained from the combined data for all the selected bugs.

When length is considered on the $\mathrm{x}$ axis :

$\mathrm{y}=-0.465 \mathrm{x}+11$

When weight is considered on the $\mathrm{x}$ axis:

$y=-0.0648 x+1.43$

Equating $(2 \mathrm{mpq}) \mathrm{x}+\mathrm{c}+[\mathrm{z} 1+\mathrm{z} 2] ; \quad(\mathrm{x}=$ number of generations passed) with regression equations

$(2 \mathrm{~m} 1 \mathrm{pq}) \mathrm{x}+\mathrm{c}+[\mathrm{z} 1+\mathrm{z} 2]=-0.465 \mathrm{x}+11$

$\mathrm{m} 1=-0.08327 ; \mathrm{C} 1=\mathrm{c}+[\mathrm{z} 2+\mathrm{z} 2]=11$

$(2 \mathrm{~m} 2 \mathrm{pq}) \mathrm{x}+\mathrm{c}+[\mathrm{z} 1+\mathrm{z} 2]=-0.0648 \mathrm{x}+1.43$

$\mathrm{m} 2=-0.004744 ; \mathrm{C} 2=\mathrm{c}+[\mathrm{z} 1+\mathrm{z} 2]=1.43$ 
mavg $=-0.004744 ; \mathrm{Cavg}=6.215$

It is known that, $\mathrm{Vt}=\mathrm{pq}(1-\exp (-\mathrm{t} / 2 \mathrm{Ne}))$; where $\mathrm{v}$ is the variance in $t$ generations

$\mathrm{pq}(1-\exp (-\mathrm{t} / 2 \mathrm{Ne}))=\{\mathrm{a} / \mathrm{b} *\{(2 \mathrm{pqmavg}) \mathrm{x}+$ Cavg $\} /$ 1.1208

$\mathrm{pq}(1-\exp (-\mathrm{t} / 2 \mathrm{Ne}))=\left\{2.7432^{*}\left\{\left(-0.004744^{*} 55.84\right) \mathrm{x}+\right.\right.$ $6.215\}\} / 1.1208$

$23.4616-1.5424 \mathrm{pq}(1-\exp (-\mathrm{t} / 2 \mathrm{Ne}))=\mathrm{x}$ where; $\mathrm{x}=$ number of generations passed

It is known that the expected time before a neutral allele becomes fixed through genetic drift, according to the Wright-Fisher model, is

Tfixed $=[-4 \mathrm{Ne}(1-\mathrm{p}) \ln (1-\mathrm{p})] / \mathrm{p}$

equating Tfixed $=\mathrm{t}$ in

$23.46161 .5424 \mathrm{pq}(1-\exp (-\mathrm{t} / 2 \mathrm{Ne}))=\mathrm{x}$

$\Rightarrow$

$23.4616-1.5424\left\{\mathrm{p}(1-\mathrm{p})\left(1-\exp \left\{2^{*}(1-\mathrm{p}) \ln (1-\mathrm{p})\right\} / \mathrm{p}\right)=\mathrm{x}\right.$

$\alpha-1.5424\{\mathrm{p}(1-\mathrm{p})(1-\mathrm{e} \boldsymbol{\beta}\}=\mathrm{x}$

where; $\mathrm{x}=$ number of generations passed

$\alpha=23.4616$

$\beta=\{2(1-p) \ln (1-p\} / p$

\section{RESULT}

Natural selection was simulated over ten filial generations by analyzing various parameters. Studying the individual plot for these parameters, it became evident that average speed had the maximum deviation which indicates major impact over survival of bugs. Therefore average speed was used as the defining character for considering survival of bugs. [2] Plotting the graph of other parameters with average speed and analyzing it as a pareto revealed that weight followed by length had maximum impact over average speed and were further studied to see the range which enabled maximum average speed. [3] Analyzing the data it became evident that short length and moderately heavy weight enabled maximum average speed. The capability analysis of the process of selection in the range of $0.6 \mathrm{~cm}-1 \mathrm{~cm}$ for length and $10 \mathrm{~g}-11.4 \mathrm{~g}$ for weight had moderately high values of $\mathrm{z}$ potential indicating high capability of the process in the above defined range. [4] Further, the selection of bugs was streamlined using the parameter of diet. Studying the observed speed in the first run, second run and then the average speed for all the bugs, the bugs fed with high fat diet showed higher speed in the second run and more symmetric average speed which enabled higher survival for these bugs. [5] Using the phenogram made for the selected bugs, most recent common ancestors were identified along with the bugs showing similar characters with recent ancestors. These bugs were then removed from the final population of bugs under study which now eliminated all bugs which had not undergone evolution. The final population was segregated into male and female bugs. Out of the male bugs an ideal bug with length $0.8 \mathrm{~cm}$ and weight $10.7 \mathrm{~g}$ and out of the female bugs an ideal bug with length $0.6 \mathrm{~cm}$ and weight $10.7 \mathrm{~g}$ was selected. The bugs showing least relative difference with ideal bugs were finally selected.[6] On continuous mapping of these selected male and female bugs over hundred seed values and plotting the frequency of selected generation over these hundred tests a final analysis was made to identify the microevolutionary force present. The microevolutionary force identified in males and females was genetic drift. In addition to that a mutation and selection trend was observed for

$\mathrm{A} 1 \mathrm{~A} 1$ and $\mathrm{A} 2 \mathrm{~A} 2$ in males and $\mathrm{A} 1 \mathrm{~A} 2$ in females.Similar results could be verified with computer models. The model showed that after 1000 generations there was a complete removal of victims 
along with the formation of a new species in males which clearly indicated that it was a slow evolutionary process showing bottleneck effect therefore along with genetic drift, the microevolutionary forces mutation and selection become evident.Similar results could be observed in females after 2000 generations. [7] The results obtained from the BSA test showed that out of the studied samples a,b,c, d, e and f. The samples e and $f$ had no protein, samples a and c which were from more recent generation had higher concentration of protein in comparison th sample $b$ and $d$ which belonged to older generations and had moderate protein concentration. Moreover, analyzing the concentration of protein in bugs and the frequency of the generations of these bugs obtained from continuous mapping revealed that the bugs with higher frequency had higher concentration of protein i.e. generation by generation protein synthesis in the body of organism increased as per the environmental factor received. Further, it could be hypothesised that change in the concentration of proteins from generation to generation supported microevolutionary forces which further brought about evolution. [8] The bugs of parent generation belonged to two different origins of Ukraine and Portugal. Selecting the fittest bug from each generation, DNA isolation was performed followed by gel electrophoresis to study adaptive radiation. Male bug from first, second, third, fifth, sixth, seventh, eighth and tenth generation and female bugs from first, second, third, seventh, eighth and ninth generation belonged to the origin Ukraine whereas the male bugs from fourth and ninth generation and female hugs from fourth, fifth, sixth and tenth generation belonged to the origin Portugal exhibiting adaptive radiation. [9] Using the above results, ratios obtained from studying Punnett Square, regression equations obtained from studying length and weight for all selected bugs and Wright-Fisher model known for examining the expected time before a neutral allele becomes fixed through genetic drift a mathematical equation linking natural selection with genetic drift and further adaptive radiation was derived.

The required equation for the above relation was

$\alpha-1.5424\{\mathrm{p}(1-\mathrm{p})(1-\mathrm{e} \boldsymbol{\beta}\}=\mathrm{x}$

where; $\mathrm{x}=$ number of generations passed

$\alpha=23.4616$

$\beta=\{2(1-p) \ln (1-p\} / p$

\section{CONCLUSION}

In light of obtained observations and results, the experiment demonstrated the simulation of natural selection using the defining criteria average speed along with length and weight over ten filial generations. These selected bugs were analyzed using phenograms, continuous mapping etc. to identify the microevolutionary forces which operated over generations and influenced the selection of the bugs.

An analysis of the concentration of proteins revealed the increase in concentration of proteins in newer and more recent generations.Further, studying the contrasting origin in the parent generation and further bugs in different generations belonging to different origins, adaptive radiation was identified. Therefore, variation in protein concentration i.e. at the macromolecular level and microevolutionary forces could be used to establish the link between selection and radiation/speciation. This relation was also mathematically derived using Punnett Square and regression models.

\section{REFERENCES}

[1]. One-parameter Semigroups for Linear Evolution Equations by Klaus-Jochen Engel and Rainer Nagel with contributions by S. Brendle, M. Campiti, T. Hahn, G. Metafune, G. Nickel, D. 
Pallara, C. Perazzoli, A. Rhandi, S. Romanelli, and R. Schnaubel Graduate Text in Mathematics 194, Springer-Verlag New York, 2000, xxi+586 pp. ISBN 0-387-98463-1

[2]. Patricia Gibert, Vincent Debat and Cameron K Ghalambor, Phenotypic plasticity, global change, and the speed of adaptive evolution, Current Opinion in Insect Science, 10.1016/j.cois.2019.06.007, (2019).

[3]. Graham J. Slater and Anthony R. Friscia, Hierarchy in adaptive radiation: A case study using the Carnivora (Mammalia), Evolution, 73, 3, (524-539), (2019).

[4]. Trina Y. Du, Sylvie C. Tissandier and Hans C. E. Larsson, Integration and modularity of teleostean pectoral fin shape and its role in the diversification of acanthomorph fishes, Evolution, 73, 2, (401-411), (2019).

[5]. Craig R. White, Dustin J. Marshall, Lesley A. Alton, Pieter A. Arnold, Julian E. Beaman, Candice L. Bywater, Catriona Condon, Taryn S. Crispin, Aidan Janetzki, Elia Pirtle, Hugh S. Winwood-Smith, Michael J. Angilletta, Stephen F. Chenoweth, Craig E. Franklin, Lewis G. Halsey, Michael R. Kearney, Steven J. Portugal and Daniel Ortiz-Barrientos, The origin and maintenance of metabolic allometry in animals, Nature Ecology \& Evolution, 10.1038/s41559019-0839-9, 3, 4, (598-603), (2019).

[6]. Daniela M. Rossoni, Bárbara M. A. Costa, Norberto P. Giannini and Gabriel Marroig, A multiple peak adaptive landscape based on feeding strategies and roosting ecology shaped the evolution of cranial covariance structure and morphological differentiation in phyllostomid bats, Evolution, 73, 5, (961-981).

[7]. Marcela Randau, Daniela Sanfelice and Anjali Goswami, Shifts in cranial integration associated with ecological specialization in pinnipeds (Mammalia, Carnivora), Royal Society Open
Science, 10.1098/rsos.190201， 6, 3, (190201), (2019).

[8]. Erik I. Svensson and David Berger, The Role of Mutation Bias in Adaptive Evolution, Trends in Ecology \& Evolution, 10.1016/j.tree.2018.01.015, 34, 5, (422-434), (2018).

[9]. Yhe softwares Gene Compiler and Plotly were used for making computer models.

\section{Cite this article as :}

Bharat Kwatra, Maanvi Mudgil, "Untangling the Mathematical Relation Between Natural Selection and Adaptive Radiation Using Macromolecules and Microevolutionary Forces", International Journal of Scientific Research in Science and Technology (IJSRST), Online ISSN : 2395-602X, Print ISSN : 23956011, Volume 7 Issue 2, pp. 313-339, March-April 2020. Available at doi : https://doi.org/10.32628/IJSRST207260 Journal URL : http://ijsrst.com/IJSRST207260 